\title{
Target Molecules of STIM Proteins in the Central Nervous System
}

\author{
Karolina Serwach and Joanna Gruszczynska-Biegala* \\ Molecular Biology Unit, Mossakowski Medical Research Centre, Polish Academy of Sciences, Warsaw, Poland
}

Stromal interaction molecules (STIMs), including STIM1 and STIM2, are single-pass transmembrane proteins that are located predominantly in the endoplasmic reticulum (ER). They serve as calcium ion $\left(\mathrm{Ca}^{2+}\right)$ sensors within the ER. In the central nervous system (CNS), they are involved mainly in Orai-mediated store-operated $\mathrm{Ca}^{2+}$ entry (SOCE). The key molecular components of the SOCE pathway are well-characterized, but the molecular mechanisms that underlie the regulation of this pathway need further investigation. Numerous intracellular target proteins that are located in the plasma membrane, ER, cytoskeleton, and cytoplasm have been reported to play essential roles in concert with STIMs, such as conformational changes in STIMs, their translocation, the stabilization of their interactions with Orai, and the activation of other channels. The present review focuses on numerous regulators, such as Homer, SOCE-associated regulatory factor (SARAF), septin, synaptopodin, golli proteins, partner of STIM1 (POST), and transcription factors and proteasome inhibitors that regulate STIM-Orai interactions in the CNS. Further we describe novel roles of STIMs in mediating $\mathrm{Ca}^{2+}$ influx via other than Orai pathways, including TRPC channels, VGCCs, AMPA and NMDA receptors, and group I metabotropic glutamate receptors. This review also summarizes recent findings

OPEN ACCESS

Edited by:

Daniele Dell'Orco,

University of Verona, Italy

Reviewed by:

Rodrigo Portes Ureshino,

Federal University of São Paulo, Brazil Francesco Moccia,

University of Pavia, Italy

*Correspondence:

Joanna Gruszczynska-Biegala jgruszczynska@imdik.pan.pl

Received: 14 October 2020 Accepted: 02 December 2020 Published: 23 December 2020

Citation:

Serwach K and

Gruszczynska-Biegala J (2020) Target Molecules of STIM Proteins in the Central Nervous System. Front. Mol. Neurosci. 13:617422. doi: 10.3389/fnmol.2020.617422 on additional molecular targets of STIM proteins including SERCA, IP 3 Rs, end-binding proteins (EB), presenilin, and CaMKII. Dysregulation of the SOCE-associated toolkit, including STIMs, contributes to the development of neurodegenerative disorders (e.g., Alzheimer's disease, Parkinson's disease, and Huntington's disease), traumatic brain injury, epilepsy, and stroke. Emerging evidence points to the role of STIM proteins and several of their molecular effectors and regulators in neuronal and glial physiology and pathology, suggesting their potential application for future therapeutic strategies.

Keywords: STIM, SOCE components, glutamate receptors, $\mathrm{Ca}^{2+}$ channels, calcium signaling, STIM regulators and effectors, store-operated calcium entry (SOCE), central nervous system

\section{INTRODUCTION}

Calcium ion $\left(\mathrm{Ca}^{2+}\right)$ is a second messenger of crucial importance to neurons as it participates in the transmission of the depolarizing signals and contributes to synaptic activity and apoptosis. Cytoplasmic $\mathrm{Ca}^{2+}$ level in neurons is regulated in a comprehensive way via the components localized in the plasma membrane (PM) such as ion channels, exchangers, and pumps, as well as the components localized in the mitochondria, endoplasmic reticulum (ER), Golgi apparatus, and nucleus (Brini et al., 2014).

Plasma membrane $\mathrm{Ca}^{2+}$ channels in neurons are divided into three major groups according to their mechanism of action: voltage-gated $\mathrm{Ca}^{2+}$ channels (VGCC), 
receptor-operated $\mathrm{Ca}^{2+}$ channels (ROC: $\alpha$-amino-3-hydroxy-5methyl-4-isoxazole propionic acid receptors [AMPARs] and $\mathrm{N}$ methyl-D-aspartate receptors [NMDARs]), and store-operated channels (SOC; Orai, transient receptor potential cation [TRPC] channels, arachidonate-regulated $\mathrm{Ca}^{2+}$ [ARC] channels) (Brini et al., 2014). In neurons, $\mathrm{Ca}^{2+}$ entry from the extracellular space is mediated via VGCCs and glutamate receptors (ionotropic: AMPAR, NMDAR, and metabotropic: mGluR) and is complemented by store-operated $\mathrm{Ca}^{2+}$ entry (SOCE) (Brini et al., 2014). Noteworthy, SOCE is the main $\mathrm{Ca}^{2+}$ source in resting neurons, while after depolarization $\mathrm{Ca}^{2+}$ influx is mediated mainly via VGCC, NMDAR and AMPAR (Brini et al., 2014). mGluR mediates both rapid transient depolarization and prolonged depolarization (Brini et al., 2014). Two systems responsible for $\mathrm{Ca}^{2+}$ extrusion from the cytoplasm to the extracellular milieu are $\mathrm{PM} \mathrm{Ca}^{2+}$ adenosine triphosphatase (PMCA) and PM Na ${ }^{+} / \mathrm{Ca}^{2+}$ exchanger (NCX). While NCX affinity to $\mathrm{Ca}^{2+}$ is low, its capacity is high. Contrary, PMCA is characterized by opposite properties (Blaustein et al., 2002; Brini and Carafoli, 2011; Brini et al., 2014).

Mitochondria are also essential components of neuronal $\mathrm{Ca}^{2+}$ toolkit. They modulate intensity and duration of $\mathrm{Ca}^{2+}$ signals following extracellular stimuli (Duszyński et al., 2006). Since they have the ability to accumulate $\mathrm{Ca}^{2+}$, they function as $\mathrm{Ca}^{2+}$ buffers. Mitochondria localized in close proximity to $\mathrm{Ca}^{2+}$ channels are exposed to high $\mathrm{Ca}^{2+}$ level and can accumulate $\mathrm{Ca}^{2+}$ efficiently. This decreases local $\mathrm{Ca}^{2+}$ level and results in depletion of $\mathrm{ER} \mathrm{Ca}^{2+}$ stores and activation of SOCE (Duszyński et al., 2006; Spät and Szanda, 2017). Special communication between mitochondria and the ER also enables $\mathrm{Ca}^{2+}$ release from the ER to mitochondria and its accumulation in the mitochondrial matrix. Increased $\mathrm{Ca}^{2+}$ concentration in the mitochondrial matrix stimulates the energy metabolism and boosts the activity of the tricarboxylic acid cycle enzymes, providing reducing equivalents to the respiratory chain and thus influencing the production of ATP (Brini et al., 2014). Calcium influx during SOCE results in mitochondrial $\mathrm{Ca}^{2+}$

Abbreviations: AD, Alzheimer's disease; AMPAR, $\alpha$-amino-3-hydroxy-5-methyl4 -isoxazolepropionic acid receptor; APP, amyloid- $\beta$ precursor protein; $\mathrm{Ca}^{2+}$, calcium ion; CaMKII, $\mathrm{Ca}^{2+} /$ calmodulin-dependent protein kinase II; CNS, central nervous system; CRAC, $\mathrm{Ca}^{2+}$ release-activated channel; DNs, dopaminergic neurons; dSTIM1, Drosophila STIM1; EB, end-binding protein; ER, endoplasmic reticulum; FAD, Familial Alzheimer's disease; HD, Huntington's disease; $\mathrm{IP}_{3}$, inositol-1,4,5-trisphosphate 3; $\mathrm{IP}_{3} \mathrm{R}, \mathrm{IP}_{3}$ receptor; KI, knockin; KO, knockout; LTD, long-term depression; LTP, long-term potentiation; MBP, myelin basic protein; mGluR, metabotropic glutamate receptor; MT, microtubule; NEUROD2, Neurogenic differentiation factor 2; NMDAR, $N$-methyl-D-aspartate receptor; nNOS, neuronal nitric oxide synthase; NPC, neural progenitor cell; OASF, Orail-activating small fragment; OPC, oligodendrocyte progenitor cell; PD, Parkinson's disease; PLA, proximity ligation assay; PLC, phospholipase C; PM, plasma membrane; PMCA, plasma membrane $\mathrm{Ca}^{2+}$ adenosine triphosphatase; PN, Purkinje neuron; POST, partner of STIM1; PS, presenilin; PSD, post-synaptic density; SARAF, SOCE-associated regulatory factor; SERCA, sarco/endoplasmic reticulum $\mathrm{Ca}^{2+}$-adenosine triphosphatase; siRNA, small-interfering RNA; SOAR, STIM-Orai-activating region; SOC, store-operated channel; SOCE, store-operated $\mathrm{Ca}^{2+}$ entry; SP, synaptopodin; STIM, stromal interaction molecule; TRPC, transient receptor potential cation channel subfamily C; TRPM, transient receptor potential cation channel subfamily M; VGCC, voltage-gated $\mathrm{Ca}^{2+}$ channel; WT, wildtype. uptake, which in turn boosts mitochondrial energy metabolism. If $\mathrm{Ca}^{2+}$ overload appears, it may cause cell apoptosis (Spät and Szanda, 2017). Thus, mitochondria link cell metabolism with $\mathrm{Ca}^{2+}$ signaling and homeostasis (Duszyński et al., 2006).

Neuronal $\mathrm{Ca}^{2+}$ signaling also appears to be pivotal in the nucleus. Cell depolarization propagates $\mathrm{Ca}^{2+}$ to the nucleus where they target the CREB transcription factor and DREAM transcriptional repressor, thereby affecting the transcription of many genes (Dick and Bading, 2010).

In neurons, the ER constitutes a vital $\mathrm{Ca}^{2+}$ storage organelle. Release of $\mathrm{Ca}^{2+}$ from the ER occurs via ryanodine receptor (RyR) and inositol-1,4,5-trisphosphate $3\left(\mathrm{IP}_{3}\right)$ receptor $\left(\mathrm{IP}_{3} \mathrm{R}\right) . \mathrm{Ca}^{2+}$ release through $\mathrm{IP}_{3} \mathrm{R}$ occurs in response to $\mathrm{mGluR}$ activation in the PM. In turn, an elevated level of cytoplasmic $\mathrm{Ca}^{2+}$ is the major trigger for $\mathrm{Ca}^{2+}$ release via $\mathrm{RyR}$ in the mechanism known as $\mathrm{Ca}^{2+}$-induced $\mathrm{Ca}^{2+}$ release (CICR) (Brini et al., 2014). The decreased $\mathrm{Ca}^{2+}$ level in the ER is refilled by SOCE.

SOCE is based on the influx of $\mathrm{Ca}^{2+}$ from the extracellular environment through channels of the PM and the replenishment of these ions in the ER when their levels decrease because of release into the cytoplasm (Blaustein and Golovina, 2001; Putney, 2003). The depletion of $\mathrm{ER} \mathrm{Ca}^{2+}$ stores is detected by stromal interaction molecules (STIMs), including STIM1 and STIM2 proteins, that are sensors of $\mathrm{Ca}^{2+}$ levels in the ER (Liou et al., 2005; Roos et al., 2005; Zhang et al., 2005). After the activation of $\mathrm{IP}_{3} \mathrm{Rs}$, the drop in $\mathrm{Ca}^{2+}$ concentration in the ER (Berridge et al., 2000) causes the oligomerization of STIM proteins and their movement toward ER-PM junctions (Liou et al., 2005; Zhang et al., 2005; Wu et al., 2006; Serwach and GruszczynskaBiegala, 2019). At these junctions, STIM proteins form complexes with proteins of $\mathrm{Ca}^{2+}$ release-activated channels (CRACs) that are formed by Orais or SOCs that consist of Orais and TRPC channels, leading to the activation of these channels (Liou et al., 2005; Mercer et al., 2006; Soboloff et al., 2006c; Liao et al., 2008; Salido et al., 2009; Saul et al., 2014; Albarran et al., 2016). Two types of $\mathrm{Ca}^{2+}$ currents are caused by $\mathrm{Ca}^{2+}$ store depletion: $\mathrm{I}_{\mathrm{CRAC}}$ (mediated by the activation of Orail and STIM1) and ISOC (involving Orail, TRPC1, and STIM1; Desai et al., 2015). Channel activation results in $\mathrm{Ca}^{2+}$ influx from the extracellular milieu to the cytoplasm (Prakriya et al., 2006), and then $\mathrm{Ca}^{2+}$ is taken to the ER by sarco/endoplasmic reticulum $\mathrm{Ca}^{2+}$-adenosine triphosphatase (SERCA) pump.

The interaction between STIM proteins and Orail is widely known to be essential for the proper function of SOCE in non-excitable cells. SOCE is a ubiquitous cell signaling pathway that is also present in many other tissues, including the rodent and human brain (Moccia et al., 2015) where it is involved in the regulation of intracellular ionic equilibrium and determines the excitability of neurons (Emptage et al., 2001; Gemes et al., 2011; Sun et al., 2014; Majewski and Kuznicki, 2015).

STIM proteins were originally described in non-excitable cells. They are now known to be present in most cells, including excitable cells, such as neurons, where STIM2 protein is predominantly expressed (Berna-Erro et al., 2009; SkibinskaKijek et al., 2009; Gruszczynska-Biegala et al., 2011; Steinbeck et al., 2011). The primary function of STIM2 in neurons was suggested to be the regulation of resting levels of $\mathrm{Ca}^{2+}$ in 
the ER and $\mathrm{Ca}^{2+}$ leakage (Gruszczynska-Biegala et al., 2011; Gruszczynska-Biegala and Kuznicki, 2013). The main function of STIM1 in neurons appears to involve the activation of SOCE (Gruszczynska-Biegala et al., 2011). Various studies have also identified STIM proteins in neuroglial cells, such as astroglia, tumor cells of astroglial origin, oligodendrocyte progenitor cells (OPCs), and microglia (Kettenmann and Bruce, 2013; Verkhratsky and Parpura, 2014; Kraft, 2015; Molnár et al., 2016). Although both STIM1 and STIM2 are expressed in astroglia, STIM1 is thought to be the more abundant isoform in these cells (Gruszczynska-Biegala et al., 2011; Kraft, 2015). Gruszczynska-Biegala et al. showed that Stim1 mRNA levels in both astroglial and neuronal cortical cultures were similar (Gruszczynska-Biegala et al., 2011).

In addition to interactions with and gating Orai, STIMs were found to recognize numerous interaction partners other than Orai. Thus, the present review focuses on the most important highly divergent target molecules of STIM proteins including positive and negative effectors and regulators in the central nervous system (CNS), mainly in neurons and glia. Recent data revealed a key role for STIM in several physiological and pathological conditions, including hypoxic/ischemic neuronal injury, traumatic brain injury (TBI), epilepsy, Alzheimer's disease (AD), Parkinson's disease (PD), and Huntington's disease (HD; Berna-Erro et al., 2009; Gemes et al., 2011; Steinbeck et al., 2011; Sun et al., 2014; Zhang et al., 2014, 2015; Popugaeva et al., 2015; Rao et al., 2015; Vigont et al., 2015; Tong et al., 2016; Czeredys et al., 2018; Serwach and Gruszczynska-Biegala, 2019). Therefore, studies of SOCE and STIM proteins may elucidate pathogenic mechanisms that are involved in the development of these diseases. Consequently, positive and negative modulators of STIM protein function or translocation may have many potential therapeutic applications. Thus, we also briefly discuss the pathophysiological significance of STIM protein interactions with their target proteins.

\section{STIM PROTEIN STRUCTURE}

STIM1 and STIM2 proteins are encoded by the STIM1 and STIM2 genes, respectively, in humans (Williams et al., 2001). They are type 1 transmembrane proteins that are localized in the ER, although STIM1 was also found in the PM (Williams et al., 2001; Liou et al., 2005; Roos et al., 2005; Keil et al., 2010). Both isoforms contain luminal and cytosolic domains (Figure 1; Soboloff et al., 2012; Moccia et al., 2015). The ER luminal Nterminal domain consists of a conserved cysteine pair, a $\mathrm{Ca}^{2+}$ binding canonical EF-hand (cEF) domain, a non- $\mathrm{Ca}^{2+}$-binding hidden EF-hand (hEF) domain, a sterile $\alpha$-motif (SAM) with one (for STIM2) or two (for STIM1) N-glycosylation sites, and a transmembrane domain (TMD). The cytosolic C-terminus includes three coiled-coil regions (CC1, CC2, and CC3) with a STIM-Orai-activating region (SOAR). The SOAR contains four $\alpha$-helices $(S \alpha 1, S \alpha 2, S \alpha 3$, and $S \alpha 4)$ and a KIKKKR sequence, which is required for the activation of Orail (Yuan et al., 2009; Yang et al., 2012). The CRAC activation domain (CAD) and Orail-activating small fragment (OASF) are both larger than the SOAR, contain a CC1 region, and activate Orail (Muik et al., 2009; Park et al., 2009). SOAR function is inhibited by

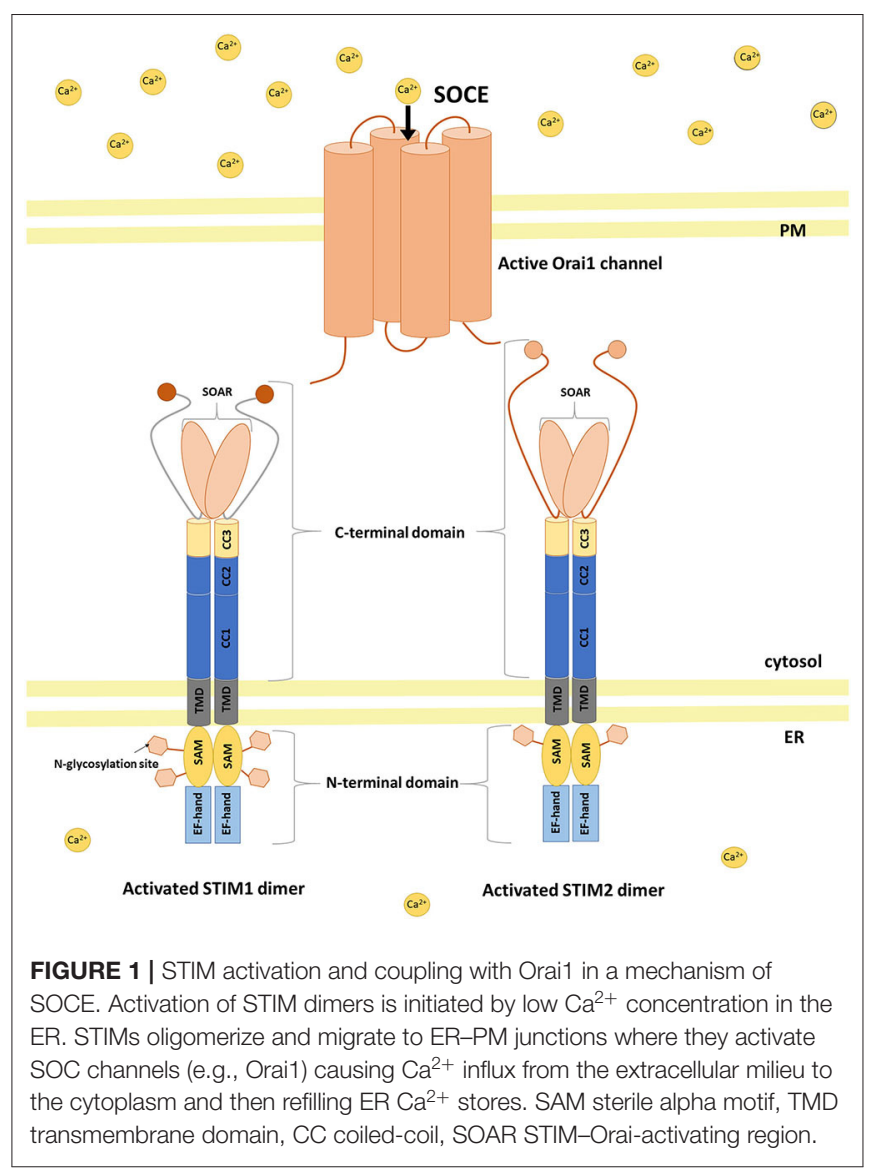

an inhibitory helix that is localized in C $\alpha 3$ (Yang et al., 2012). Downstream of SOAR is an acidic inhibitory domain (ID) that also mediates the fast $\mathrm{Ca}^{2+}$-dependent inactivation of Orail (Lee et al., 2009). The C-terminus tail of STIM proteins also contains a proline/serine-rich (PS) domain, a microtubule-interacting domain, and a polybasic lysine-rich domain that is responsible for phospholipid interaction in the PM (Soboloff et al., 2012).

STIM1 and STIM2 proteins diverge significantly within the C-terminus (Figure 1). Strong evidence indicates that STIM proteins associate in vivo, and these interactions may be mediated by an association between CC regions of C-terminal ends of these proteins (Soboloff et al., 2006a). Notably, STIM2 has lower affinity for $\mathrm{Ca}^{2+}$ sensing compared with STIM1 because of three amino acid substitutions in cEF that allow STIM2 to detect smaller decreases in $\mathrm{Ca}^{2+}$ inside the ER lumen (Brandman et al., 2007; Hoth and Niemeyer, 2013).

\section{STIM PROTEINS IN THE PHYSIOLOGY OF NEURONS, AUTOPHAGY, AND NEURODEGENERATIVE DISEASES}

\section{Physiology}

By activating neuronal SOCE, STIM proteins play a pivotal role in the physiology of neurons (Serwach and GruszczynskaBiegala, 2019). In neurons deprived of STIM2, the amount of mushroom dendritic spines, which are vital for memory storage, was decreased (Sun et al., 2014; Garcia-Alvarez et al., 2015; Yap 
et al., 2017). STIM2 also co-localizes with $\mathrm{Ca}^{2+} /$ calmodulindependent protein kinase II (CaMKII) in dendritic spines and regulates its phosphorylation (Yap et al., 2017). STIM2-mediated SOCE sustained CaMKII activation and thus is important in the maintenance of dendritic spines. STIM2-Orai2-TRPC6 complexes regulate SOCE in mice hippocampal synapses and thus influence the number of dendritic spines. Orai-STIM2 complexes play an essential role in the formation of new synapses (Korkotian et al., 2017). All these studies demonstrate a vital role of STIM-mediated SOCE in both formation and maintenance of mushroom spines, and suggest its role in synaptic plasticity. Neuronal SOCE indeed takes part in longterm potentiation (LTP) and long-term depression (LTD), processes responsible for memory and learning (Serwach and Gruszczynska-Biegala, 2019). In Purkinje neurons (PNs), slow excitatory post-synaptic currents (EPSCs) were the result of TRPC3 activity. Lack of STIM1 resulted in no ER $\mathrm{Ca}^{2+}$ release and slow EPSCs (Hartmann et al., 2014). Thus, STIM1 is considered to refill the dendritic $\mathrm{ER} \mathrm{Ca}^{2+}$ stores only under resting conditions. In resting cells, STIM1-mediated SOCE also caused an ubiquitination and degradation of Sp4 transcription factor (Lalonde et al., 2014). These results underlie an essential homeostatic function of STIM1-mediated SOCE in resting neurons.

\section{Autophagy}

Autophagy is stimulated in response to various types of cellular stress, including ER stress, oxidative stress, starvation of nutrients and growth factors, hypoxia and mitochondrial damage (Kroemer et al., 2010). Moderate ER stress can improve the ability of the ER to process unfolded or misfolded proteins and maintain cell survival. However, if the stress is prolonged or extensive, homeostasis within the cell is disrupted leading to apoptosis, which is primarily mediated by $\mathrm{Ca}^{2+}$ overload, or autophagy (Berridge et al., 2000; Bernales et al., 2006; Ding et al., 2007; Høyer-Hansen and Jäättelä, 2007). Autophagy, e.g., caused by oxygen and glucose deprivation and reoxygenation, may maintain cellular homeostasis, but its excessive level may lead to autophagic neuronal necrosis and apoptosis (Ahsan et al., 2019; Zhou et al., 2019). Differences in cytosolic $\mathrm{Ca}^{2+}$ levels associated with autophagy and apoptosis have been demonstrated in several cell lines. Calcium is mostly considered as an activator of autophagy, but there are some reports that $\mathrm{Ca}^{2+}$ suppresses autophagy (Høyer-Hansen et al., 2007; Cárdenas et al., 2010; Law et al., 2010; Parys et al., 2012; Wong et al., 2013).

However, the role of STIM1/Orail in autophagy and apoptosis in the CNS is still unclear, and there is not much work on the subject, thus underscoring the need for further research in this field. Proteasome inhibitors MG-132 and LA promoted the autophagy-mediated degradation of STIM1 and STIM2 and thus reduced SOCE in neurons (Kuang et al., 2016). The opposite is true in HEK293 cells where the stability of STIM1 was not affected by proteasome inhibitors, although thapsigargininduced surface levels of STIM1 and SOCE were increased in cells pretreated with MG-132 (Keil et al., 2010). These differences are likely to be due to the different conditions for treating cells with protease inhibitors and may also exist depending on the type of cells used. Further, Kondratskyi et al. demonstrated that, in prostate cancer cells, SOCE inhibitor (ML-9) stimulates autophagosome formation and inhibits autophagosome degradation independent of SOCE and STIM1 (Kondratskyi et al., 2014). On the other hand, in prostate cancer cells (DU145 and PC3), resveratrol has been proposed to induce autophagy by regulating the function of STIM1 and then SOCE. Indeed, STIM1 overexpression restores resveratrol-induced reduction of SOCE as well as autophagic cell death induced by ER stress (Selvaraj et al., 2016). In line with this, STIM1 and SOCE have been shown to positively regulate oxidized low-density lipoprotein-induced autophagy in endothelial progenitor cells (Yang et al., 2017). Recently, hypoxia-induced $\mathrm{Ca}^{2+}$ release from the ER in neuron-like PC12 cells was modulated by STIM1/Orai1 (Hu et al., 2020). In addition, STIM1/Orail signaling induced by $\alpha 2$-adrenergic receptor agonist, dexmedetomidine, following hypoxia was mediated by a decrease of $\left[\mathrm{Ca}^{2+}\right]_{i}$, leading to a reduction of autophagy. The results suggest that dexmedetomidine may have neuroprotective effects against oxidative stress, autophagy, and neuronal apoptosis after oxygen-glucose deprivation and reoxygenation injury through modulation of $\mathrm{Ca}^{2+}$-STIM1/Orai1 signaling (Hu et al., 2020). In turn, STIM1 has been shown to be not essential in hypoxia-mediated autophagy in both SHSY5Y and HSG cells (Sukumaran et al., 2015). The available data suggest that STIMs influence autophagy differently depending on cell type and triggers of autophagy.

\section{Neurodegenerative Diseases}

Dysregulation of neuronal SOCE and changes in STIM expression levels are associated with various pathological conditions of the CNS such as hypoxic/ischemic neuronal injury, TBI, epilepsy, AD, PD and HD (Figure 2; Serwach and Gruszczynska-Biegala, 2019). Many studies have demonstrated the role of STIM2 in hypoxic/ischemic neuronal injury (Soboloff et al., 2006b; Vig et al., 2006; Berna-Erro et al., 2009). Hippocampal neurons, both in slices and in culture, showed reduced ER $\mathrm{Ca}^{2+}$ level during hypoxia, and STIM2 reduced $\mathrm{Ca}^{2+}$ overload during ischemic challenge (Berna-Erro et al., 2009). Stim 2 knockout (KO) mice were better protected against cerebral ischemia (Berna-Erro et al., 2009). Thus, it seems that the absence of STIM2 may potentially constitute a protective strategy against stroke. STIM1 and STIM2 have also been implicated in epilepsy as they are up-regulated both in CA1 and CA3 regions of chronic epileptic mice (Steinbeck et al., 2011). Nonselective SOCE inhibitors rhythmized epileptic burst activity in epileptic hippocampal slices, suggesting that SOCE blockage may potentially bring positive effect in patients with epilepsy. STIM2 has also been shown to be overexpressed after TBI (Rao et al., 2015). The downregulation of STIM2 improved neuronal survival in models of TBI, decreasing neuronal apoptosis and preserving neurological function by alleviating mitochondrial disfunction and $\mathrm{Ca}^{2+}$ overload. STIM2 downregulation not only decreased $\mathrm{Ca}^{2+}$ release from the ER, but also reduced SOCE and dropped mitochondrial $\mathrm{Ca}^{2+}$ level, restoring its morphology and function. Downregulation of STIM2 has a neuroprotective effect and may be a target in TBI treatment (Rao et al., 2015). Dysregulation of SOCE also contributes to 


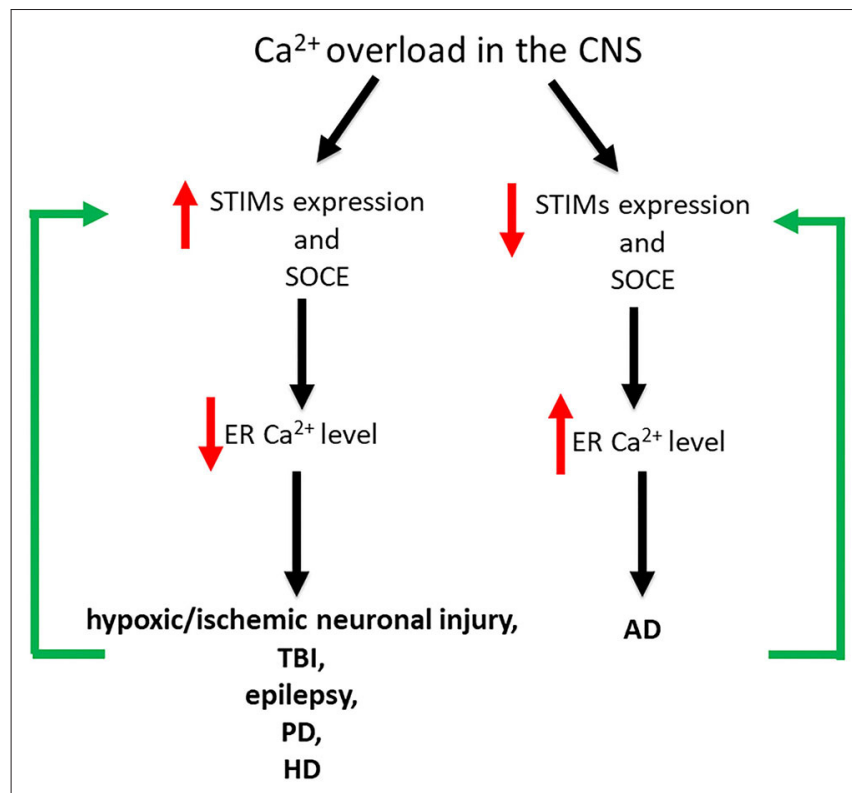

FIGURE 2 | Impact of $\mathrm{Ca}^{2+}$ overload on STIM expression level and SOCE in the development of CNS disorders. In hypoxic/ischemic neuronal injury, TBI, epilepsy, PD, and $\mathrm{HD} \mathrm{Ca}^{2+}$ overload is associated with increased STIM expression level and SOCE and thus decreased ER Ca ${ }^{2+}$ level. Contrary, in AD $\mathrm{Ca}^{2+}$ overload results in decreased STIM expression level and SOCE and thus increased ER $\mathrm{Ca}^{2+}$ level. SOCE blockage and STIM downregulation seem to be neuroprotective in hypoxic/ischemic neuronal brain injury, epilepsy, TBI, HD, and PD, while in AD increased expression level of STIMs and SOCE enhancement appear to be neuroprotective.

PD. A neurotoxin, which mimics PD in vitro, decreased level of TRPC1 and its interaction with STIM1, thus increasing neuronal death. Pharmacological inhibition of SOCE appears to be neuroprotective representing a potential target for $\mathrm{PD}$ drug discovery (Pchitskaya et al., 2018). In HD transgenic mice, over-activation of synaptic SOCE and enhancement of STIM2 expression resulted in the disruption of dendritic spines. STIM2 knockdown has been shown to normalize SOCE and prevent loss of dendritic spines. It seems that pharmacological modulation of SOCE and its components have neuroprotective effects in HD patients. On the other hand, in mice models of $\mathrm{AD}$, impairment of SOCE and reduction of synaptic STIM2 proteins contributed to the destabilization of dendritic spines (Sun et al., 2014; Zhang et al., 2016). Since stabilization of dendritic spines is considered to prevent memory loss in AD patients, the modification of STIM proteins and SOCE may be a potential therapeutic target in the treatment of memory loss in these patients.

Changes in neuronal SOCE may vary among pathological states of the CNS. SOCE blockage and STIM downregulation seem to be neuroprotective in hypoxic/ischemic neuronal brain injury, epilepsy, TBI, HD and PD, while in AD STIMs and SOCE appear to be neuroprotective (Figure 2).

Misfolded proteins and the associated ER stress are common features of some neurodegenerative diseases such as $\mathrm{PD}, \mathrm{AD}$ and HD. These properties can further induce autophagy or apoptosis in neurons (Ghavami et al., 2014; Remondelli and Renna, 2017). Given the high sensitivity of neurons to ER $\mathrm{Ca}^{2+}$ store disturbances, STIM and SOCE have been proposed as potential targets for neuroprotection by reversing ER and mitochondrial stress-induced damage. Interestingly, blockade of SOCE reduced apoptosis mediated by oxidative stress in hippocampal neuronal HT-22 cells (Rao et al., 2013). Hawkins et al. demonstrated that in lymphocyte cells oxidative stress favors STIM1 trafficking and puncta formation, which confirms that STIM1 is regulated by the redox state (Hawkins et al., 2010). In turn, the formation of the STIM1 puncta, their translocation to the PM and the subsequent SOCE in HEK cells were disrupted by mitochondrial depolarization in mitofusin 2 dependent manner. These effects have been shown to be overcome by overexpression of STIM1 (Singaravelu et al., 2011). Consequently, STIM1 in 401L neuroblastoma cells provided protection against ER stress and mitochondrial oxidative stress causing cell death (Zhang and Thomas, 2016). Experiments performed on embryonic fibroblasts also reported that STIM1 rescue protected from oxidative stress and enabled cell survival by impairing the translocation of the apoptosis-inducing factor into the nucleus (Henke et al., 2012). All these findings suggest that STIM may indeed provide protection against cell death mediated by the ER and oxidative stress, which often precede neurodegeneration.

\section{STIM-BINDING $\mathrm{Ca}^{2+}$ CHANNELS}

According to the current state of knowledge, neuronal STIM proteins regulate both CRAC and SOC. Other channel proteins, such as L-type voltage-gated $\mathrm{Ca}^{2+}$ channels (VGCCs; $\mathrm{Ca}_{\mathrm{V}} 1.2$, $\mathrm{Ca}_{\mathrm{v}} 1.3$ ) and receptor/ligand-activated $\mathrm{Ca}^{2+}$ channels (AMPARs and NMDARs), couple or engage in an interplay with STIMs in the CNS in a modulatory way as part of SOCE signaling (Figure 3).

\section{Orai}

As mentioned above, emptying $\mathrm{ER} \mathrm{Ca}^{2+}$ stores causes STIM protein oligomerization and translocation of the oligomers toward the PM where they form complexes, known as puncta, with an $\mathrm{Ca}^{2+}$ selective ion channel protein, Orail. We previously showed that the depletion of $\mathrm{Ca}^{2+}$ from the ER by thapsigargin, a selective SERCA inhibitor, increased the number of puncta-like structures with Yellow Fluorescence Protein (YFP)-STIM1 and Orail but not those with YFPSTIM2 and Orai1 (Klejman et al., 2009; Gruszczynska-Biegala et al., 2011). In contrast, a reduction of extracellular $\mathrm{Ca}^{2+}$ levels with ethylene glycol-bis( $\beta$-aminoethyl ether)- $N, N, N^{\prime}, N^{\prime}$ tetraacetic acid (EGTA) triggered the puncta formation of both YFP-STIM1/Orai1 and YFP-STIM2/Orai1. Other results showed that endogenous STIM1 and STIM2 can interact with Orai1, which was observed in a co-immunoprecipitation assay and in situ proximity ligation assay (PLA; Gruszczynska-Biegala and Kuznicki, 2013). The higher association between endogenous STIM2 and Orail in cortical neurons occurred in the presence of BAPTA-AM, membrane permeable $\mathrm{Ca}^{2+}$ chelator, and in a low- $\mathrm{Ca}^{2+}$ medium but not in the presence of thapsigargin. When SOCE was induced, the greatest number of PLA signals that corresponded to integrated STIM1 and Orail puncta was visible. The interaction between them was quantified and correlated well with the number of exogenous complexes that formed under 


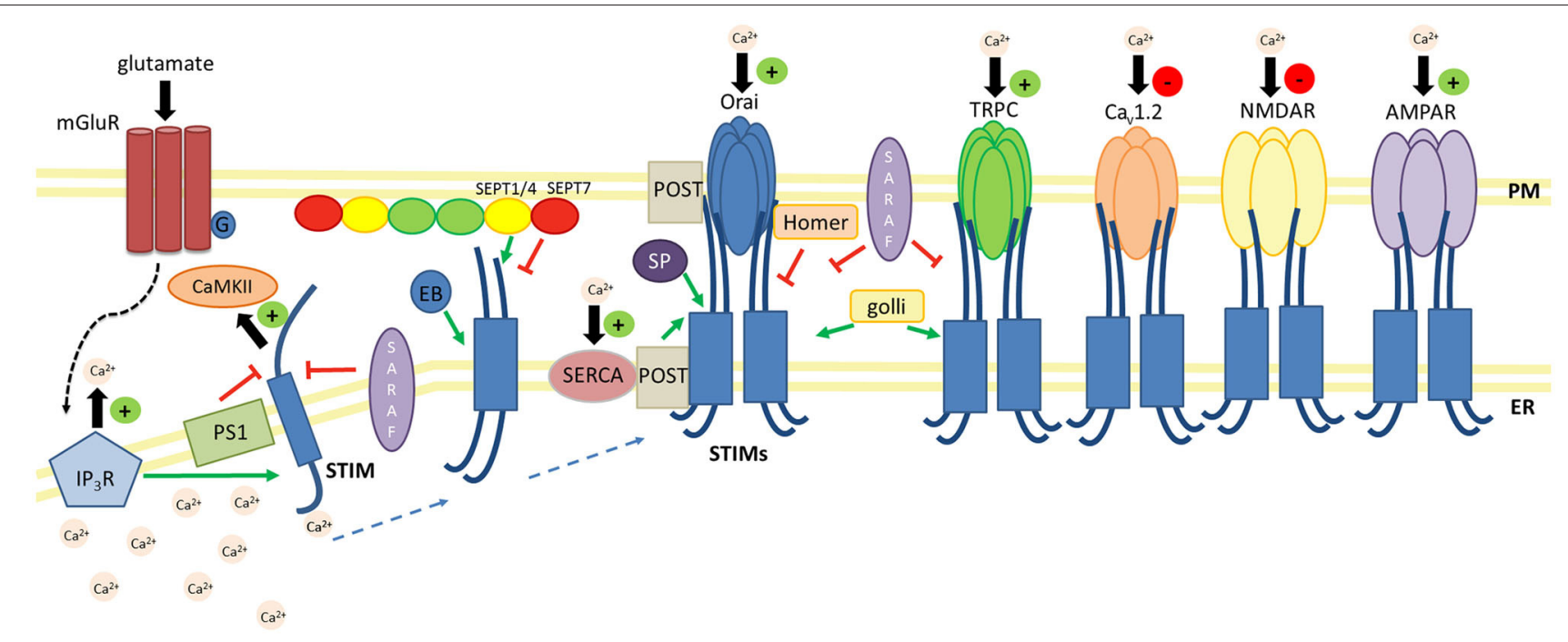

FIGURE 3 | Schematic overview of key regulators and effectors of STIM proteins in the CNS. Negative regulators (- |): SARAF prevents STIM1 activation and inhibits the STIM1-Orai association. SARAF silencing increases TRPC1-mediated $\mathrm{Ca}^{2+}$ entry. The PS1- $\gamma$-secretase complex cleaves the STIM1 transmembrane domain. Homer1a dissociates the STIM1-Orai1 complex. Lower dSEPT7 expression increases the amount of dSTIM-dOrai clusters. Positive regulators ( $\rightarrow$ ): The glutamate-mediated activation of mGluRs results in $\mathrm{Ca}^{2+}$ release from ER stores via IP $\mathrm{P}_{3} \mathrm{Rs}$ and activates STIM-Orai coupling. EB3 forms complexes with STIM2, which promotes the formation of mushroom spines in hippocampal neurons. SEPT1/4 regulates the number of ER-PM junctions and enhances STIM1-Orai1 interactions. The STIM1-POST complex binds to SERCA and promotes ER Ca ${ }^{2+}$ refilling. Golli proteins interact with STIM1 and TRPC1 and thus enhance SOCE. SP interacts with STIM and Orai and determines synaptic plasticity. Positive effectors (+): STIM proteins increase Ca ${ }^{2+}$ influx via Orai, TRPC, and AMPARs. STIM2-mediated SOCE activates CaMKII and thus stabilizes mushroom spines. Negative effectors (-): STIM proteins decrease Ca ${ }^{2+}$ influx via L-type VGCCs and NMDARs.

the same conditions (Klejman et al., 2009; Gruszczynska-Biegala et al., 2011; Gruszczynska-Biegala and Kuznicki, 2013).

We can conclude that STIM1 and STIM2 can activate Orai1 channels and play different roles in neuronal SOCE (BernaErro et al., 2009; Klejman et al., 2009; Keil et al., 2010; Gruszczynska-Biegala et al., 2011; Gruszczynska-Biegala and Kuznicki, 2013; Sun et al., 2014; Majewski and Kuznicki, 2015). In rat cortical neurons, STIM1 mainly forms complexes with Orai1 and activates SOCE only after $\mathrm{Ca}^{2+}$ is completely emptied from the ER. This demonstrates its role in maintaining the level of $\mathrm{Ca}^{2+}$ in the ER. In contrast, STIM2 forms a hetero-complex with Orail to allow the regulation of resting intracellular $\mathrm{Ca}^{2+}$ levels and activation of constitutive $\mathrm{Ca}^{2+}$ influx after a slight decrease in $\mathrm{Ca}^{2+}$ levels in the ER.

In the rat cortex, SOCE is mainly mediated by Orail-STIM1 complexes. In the mouse brain, in contrast, it is triggered either by Orai2 and STIM2 (cortex and hippocampus; Berna-Erro et al., 2009; Sun et al., 2014) or by Orai2 and STIM1 (cerebellum; Hartmann et al., 2014). Likewise, STIM2-gated SOC channels in dendritic mushroom spines are formed by a complex of Orai2 and TRPC6 (Zhang et al., 2016; Popugaeva et al., 2020). In turn, both STIM1 and STIM2 are involved in SOCE in sensory neurons in dorsal root ganglia. Moreover, both Orai1 and Orai3 contribute to SOCE in these neurons, where they may form homomultimers to mediate SOCE (Wei et al., 2017).

In addition to a canonical function as an $\mathrm{ER} \mathrm{Ca}^{2+}$ level refilling toolkit, STIM1-Orail-mediated SOCE was also shown to regulate gene expression and proliferation in mouse and human neural progenitor cells (NPCs) and thus is thought to be a key regulator of neurogenesis in mammalian cells (Somasundaram et al., 2014; Gopurappilly et al., 2018). The knockdown of Orail or STIM1 diminishes SOCE in NPCs. SOCE is not observed in NPCs from transgenic mice that lack Orail or STIM1 or in knock-in (KI) mice that express a Orail mutant. The deletion or suppression of STIM1 and Orail diminishes the proliferation of embryonic and adult NPCs both in vitro and in vivo in the subventricular zone (SVZ) in the adult mouse brain (Somasundaram et al., 2014). Domenichini et al. showed that SOCE in SVZ cells is mediated not only by STIM1 and Orail but also by TRPC1 (Domenichini et al., 2018). The pharmacological blockade of this process in mouse SVZ cells decreases proliferation and impairs selfrenewal by shifting the type of cell division from symmetric to asymmetric, thereby reducing stem cell population (Domenichini et al., 2018). In human NPCs, SOCE has been shown to be significantly attenuated by the short-hairpin RNA/micro RNA targeting of STIM1 (Gopurappilly et al., 2018). Gopurapilly et al. investigated global gene expression in human NPCs with STIM1 knockdown and showed that signaling pathways that are associated with DNA replication and cell proliferation were downregulated, whereas post-synaptic cell signaling was upregulated in these cells (Gopurappilly et al., 2018). To understand the functional relevance of these gene expression alterations, these authors also measured the self-renewal capacity of NPCs with STIM1 knockdown and found a substantially smaller neurosphere size and number and a decrease in differentiation toward cells with a neuronal lineage. These findings demonstrate that STIM1-mediated SOCE in human 
NPCs regulates gene expression alterations, which is likely to modulate the differentiation and self-renewal of NPCs (Gopurappilly et al., 2018).

STIM1 and ORAI1 have been shown to be involved in $\mathrm{Ca}^{2+}$ signaling in both astroglia and glioblastoma cells. The knockdown of both STIM1 and Orail or Orail alone resulted in a reduction of SOCE in rat astrocytes (Moreno et al., 2012). The silencing of STIM1 or Orail was shown to reduce SOCE and CRAC currents in human glioblastoma cells (Motiani et al., 2013). Surprisingly, Ronco et al. found that Orai3 but not Orail is a dominant Orai homolog in astroglia (Ronco et al., 2014). Recent studies ascribed a role to STIM1, Orai1, and Orai3 in astroglial SOCE (Gao et al., 2016; Kwon et al., 2017). Additionally, the activation of SOCE in spinal astroglia promotes the production of proinflammatory cytokines, such as tumor necrosis factor $\alpha$ (TNF- $\alpha$ ) and interleukin-6 (IL- 6 ). The production of TNF- $\alpha$ and IL- 6 was decreased by the knockdown of Orail or STIM1 (Gao et al., 2016). Interestingly, Orail and STIM2 knockdown minimized lipopolysaccharide (LPS)-induced TNF- $\alpha$ and IL-6 production (Gao et al., 2016). This research may provide a basis for assessing SOCE and its components for the treatment of chronic pain and other neurological diseases that are associated with astroglial overactivation.

Studies that focused on the optic nerve in mice identified Orail and STIM1 but not STIM2 in astrocytes, whereas Orai1, STIM1, and STIM2 in oligodendrocytes suggested that STIM1 may be localized in the cell soma, and STIM2 may be localized in myelin (Papanikolaou et al., 2017).

In cultured rat microglia, SOCE is mediated by Orai channels rather than TRPC channels (Ohana et al., 2009). Siddiqui et al. identified high levels of Orail and STIM1 in microglial podosomes, structures that are responsible for cell motility (Siddiqui et al., 2012). Another research group confirmed the expression of STIM1, Orai1, Orai2, and Orai3 in cultured mouse microglia and showed that the downregulation of STIM1 and Orail reduced SOCE in these cells (Heo et al., 2015). Michaelis et al. studied the role of Orail and STIM proteins in microglia using cells that were obtained from knockout mice (Michaelis et al., 2015). The results showed that SOCE was reduced in the absence of Orail or STIM proteins. SOCE was nearly absent in Stim $1^{-/-}$microglia and substantially reduced in Orai1 ${ }^{-/-}$microglia, whereas a less pronounced effect was observed in Stim2 $2^{-1-}$ microglia (Michaelis et al., 2015). Orail and STIM1 appear to be major components of microglial SOCE, and STIM2 is also a constituent of this signaling pathway in these cells (Kraft, 2015). Interestingly, recent studies showed that STIM1- and Orail-mediated SOCE regulate phagocytic activity and cytokine release in primary murine microglia (Heo et al., 2015). Phagocytic activity, as well as LPS stimulationmediated proinflammatory cytokine release (e.g., TNF- $\alpha$ and IL6), was inhibited by SOCE inhibitors and STIM1 and Orai1 knockdown (Heo et al., 2015). This research suggests that STIM1 may be a new regulatory target for the prevention of an excessive proinflammatory response of microglia in neurodegenerative disorders.

\section{TRPC Channels}

In addition to Orai activation, STIM proteins may cause $\mathrm{Ca}^{2+}$ influx via TRPC channels, which are found in cells from all regions of the brain and spinal cord, with high TRPV (TRP channel subfamily V), TRPC, and TRPM (TRP channel subfamily M) expression (Verkhratsky et al., 2014). Nevertheless, TRPC1 and Orail activation is mediated by different STIM1 domains. TRPC1 is involved in SOCE, but like the other TRPC channels, it is unable to generate a current that resembles $\mathrm{Ca}^{2+}$-selective I CRAC (Albarrán et al., 2016). TRPC1 function depends on Orail-mediated $\mathrm{Ca}^{2+}$ influx, which triggers the recruitment of TRPC1 into the PM, where it is activated by STIM1. TRPC1 is thought to modify the initial $\mathrm{Ca}^{2+}$ signal that is caused by Orail activation (Ambudkar et al., 2017).

TRPC1-mediated SOCE is essential for neuronal survival (Wang and Poo, 2005; Bollimuntha et al., 2006; Selvaraj et al., 2012). The STIM1-TRPC1 interaction is thought to be neuroprotective in both in vitro and in vivo models of $\mathrm{PD}$ (Selvaraj et al., 2012; Sun et al., 2017, 2018). In the human SH-SY5Y neuroblastoma cell line, SOCE mainly depends on the activation of TRPC1. A neurotoxin that caused the selective loss of dopaminergic neurons (DNs) in the substantia nigra pars compacta (SNpc) decreases TRPC1 expression, the TRPC1STIM1 interaction, and SOCE but not Orai expression (Selvaraj et al., 2012). TRPC1 overexpression prevents the neurotoxinmediated loss of SOCE and decreases ER $\mathrm{Ca}^{2+}$ levels and the unfolded protein response (UPR). Additionally, TRPC1mediated $\mathrm{Ca}^{2+}$ entry activates the neuroprotective AKT pathway. STIM1 or TRPC1 but not TRPC3 silencing increases the UPR. Consistent with these results, $\operatorname{Tr} p c 1^{-/-}$mice have a higher UPR and lower number of DNs, similar to PD patients. The overexpression of TRPC1 in mice increased DN survival after neurotoxin treatment.

STIM1 was also shown to inactivate $\mathrm{Ca}^{2+}$ entry via VGCCs, which is detrimental to DNs. Thus, the STIM1TRPC1 interaction was thought to inhibit $\mathrm{Ca}^{2+}$ influx via VGCC channels, thereby protecting DNs (Selvaraj et al., 2012). Subsequent research showed that TRPC1 regulates L-type VGCCs in SNpc neurons (Sun et al., 2017). The STIM1-TRPC1 interaction after store depletion reduced DN activity in wildtype (WT) but not $\operatorname{Tr} p c 1^{-/-}$mice. In $\operatorname{Tr} p c 1^{-/-} \mathrm{SNpc}$ neurons, Ltype VGCC Ca ${ }^{2+}$ currents increased, STIM1- $\mathrm{Ca}_{\mathrm{V}} 1.3$ interactions were attenuated, and the number of DNs decreased. After TRPC1 activation, L-type $\mathrm{Ca}^{2+}$ currents and $\mathrm{Ca}_{\mathrm{v}} 1.3$ opening probability decreased, whereas they increased after STIM1/TRPC1 silencing. Additionally, store depletion increased the $\mathrm{Ca}_{\mathrm{v}} 1.3$-TRPC1STIM1 association. TRPC1 appears to suppress $\mathrm{Ca}_{\mathrm{v}} 1.3$ activation proving that STIM1 is essential for DN survival (Sun et al., 2017).

Sun et al. showed that mesenchymal stem cell (MSC)-derived DNs, similar to native neurons, utilize TRPC1-mediated SOCE (Sun et al., 2018). Similar to SH-SY5Y cells, neurotoxin treatment in MSC-derived DNs decreased TRPC1 expression and SOCE. TRPC inhibition alleviated dopamine release and MSC-derived $\mathrm{DN}$ viability. These results indicate that $\mathrm{ER} \mathrm{Ca}^{2+}$ levels that are maintained by TRPC1-mediated SOCE are neuroprotective. 
Neurotoxin exposure may cause alterations of SOCE and TRPC1mediated $\mathrm{Ca}^{2+}$ homeostasis that may further induce ER stress and the UPR, leading to neurodegeneration. These results demonstrate that MSC-derived DNs are similar to native DNs, which potentially broadens the prospect of their usage for regenerative therapy in PD patients (Sun et al., 2018).

STIM1 and TRPC have also been shown to be involved in SOCE in astroglia. Antisense oligonucleotides that targeted the $\operatorname{Tr} p c 1$ gene reduced SOCE in murine astrocytes (Golovina, 2005). An anti-TRPC1 antibody lessened SOCE in rat astrocytes (Malarkey et al., 2008). In spinal astrocytes, SOCE was predominantly subserved by TRPC3 (Miyano et al., 2010). Some studies ascribed these differences between Orai- and TRPC-mediated SOCE to the stage of astroglial development, suggesting that SOCE in immature and maturating astroglia is predominantly mediated by Orai, whereas SOCE in mature cells is predominantly mediated by TRPC1 (Kettenmann and Bruce, 2013; Verkhratsky and Parpura, 2014). Another study speculated that Orai1 and Orai3 are expressed in astroglial cells with abundant SOCE, whereas TRPC1 is restricted to astroglia where this process is attenuated (Kwon et al., 2017). STIM proteins, Orail, and TRPM3 were identified as constituents of SOCE in astrocytes and oligodendrocytes of the mouse optic nerve (Papanikolaou et al., 2017). The developmental downregulation of Orail is consistent with TRPC channels as major components of mature astrocytes and oligodendrocytes, suggesting a potential role for Orai/STIM SOCE in immature glia and TRPM3 in mature glia (Verkhratsky et al., 2014).

Müller glia, a type of retinal glial cell, expresses STIM1 and requires the synergistic activation of both TRPC1 and Orai channels. The precise mechanism by which Orai and TRPC1 are activated by STIM1 has not been ascertained in these cells (Molnár et al., 2016).

\section{VGCCs}

VGCCs are $\mathrm{Ca}^{2+}$ channels that are present primarily in electrically excitable cells (as neurons), known as transducers of electrical activity that enable $\mathrm{Ca}^{2+}$ influx in response to subthreshold depolarizing stimuli or action potentials (Harraz and Altier, 2014). They are assembled from the pore-forming $\alpha 1$ subunit and accessory $\beta$ and $\alpha 2 \delta$-like subunits (Heine et al., 2020). Various isoforms of the $\alpha 1$ subunit differ in voltage and $\mathrm{Ca}^{2+}$ sensitivity, which defines the specific kinetic properties of the channel. Thus, VGCCs are classified into low and high voltage-activated. The accessory $\beta$ and $\alpha 2 \delta$-like subunits play a role in membrane trafficking and the modulation of kinetic properties of high voltage-activated $\mathrm{Ca}^{2+}$ channels (Campiglio and Flucher, 2015; Brockhaus et al., 2018). $\alpha 2 \delta$-like subunit and chemotaxis receptor domain containing 1 (Cachd1) alter the kinetic properties and surface expression of VGCCs (Cottrell et al., 2018; Dahimene et al., 2018). Cachd1 also impacts the gating and trafficking of low voltage-activated $\mathrm{Ca}^{2+}$ channels (Cottrell et al., 2018).

In excitable cells, VGCCs are the main route of $\mathrm{Ca}^{2+}$ entry in response to depolarizing stimuli. The main VGCC subtype that is present in neuronal, cardiac, and smooth muscle cells is $\mathrm{Ca}_{\mathrm{v}} 1.2$, whereas $\mathrm{Ca}_{\mathrm{v}} 1.3$ is the predominant subtype in DNs
(Sun et al., 2017). Both VGCC subtypes were shown to be suppressed by STIM1 (Park et al., 2010; Wang et al., 2010; Harraz and Altier, 2014; Sun et al., 2017, 2018). Two independent studies of excitable cells showed that $\mathrm{ER} \mathrm{Ca}^{2+}$ store depletion alleviates depolarization-mediated $\mathrm{Ca}_{\mathrm{v}} 1.2$ activity, whereas the $\mathrm{Ca}_{\mathrm{V}} 1.2$ response increases after functional impairments in STIM1 (Park et al., 2010; Wang et al., 2010). STIM1-Ca 1.2 interactions are directly mediated by the SOAR domain (i.e., the same domain that activates SOCs) of STIM1 and C-terminus of the $\mathrm{Ca}_{\mathrm{V}} 1.2 \alpha 1$ subunit (Harraz and Altier, 2014; Pascual-Caro et al., 2018). The influence of STIM1 on VGCCs is also associated with an increase in channel internalization from the PM. Despite reporting similar results, two studies suggested different inhibitory mechanisms. Park et al. proposed a mechanism that involves the attenuation of VGCC expression, whereas Wang et al. suggested a potential role for Orail in the inhibitory STIM1-Ca 1.2 interaction because the simultaneous inhibition of both Orail and STIM1 was necessary to suppress $\mathrm{Ca}_{\mathrm{v}} 1.2$ activity (Park et al., 2010; Wang et al., 2010).

The ability of STIM1 to regulate Orail and $\mathrm{Ca}_{\mathrm{v}} 1.2$ is tissue specific. STIM1 appears to stimulate SOCE in non-excitable cells and inhibit VGCCs in excitable cells (Harraz and Altier, 2014). STIM1 was also shown to control the plasticity of Ltype VGCC-dependent dendritic spines (Dittmer et al., 2017; Sather and Dittmer, 2019). The activation of neuronal STIM1 induces changes in the ER structure, which depends on L-type VGCCs. The NMDAR activation of L-type VGCCs triggers $\mathrm{Ca}^{2+}$ release from the ER, which in turn causes STIM1 aggregation and its coupling with L-type VGCCs and then inhibits the activation of this channel, thus increasing ER spine content and stabilizing mushroom spines (Dittmer et al., 2017). STIM1 deficiency is associated with $\mathrm{AD}$ and triggers SH-SY5Y cell death by upregulating $\mathrm{Ca}_{\mathrm{v}} 1.2$ (Pascual-Caro et al., 2018). Thus, STIM1 KO cells may constitute an in vitro model to study the pathogenesis of $\mathrm{AD}$ and may be useful for understanding the role of STIM1 in neurodegeneration. In turn, as mentioned in section TRPC Channels above, TRPC1 in DNs facilitates STIM1$\mathrm{Ca}_{\mathrm{v}} 1.3$ interactions to suppress $\mathrm{Ca}_{\mathrm{v}} 1.3$ activity, thereby reducing apoptosis and protecting DNs against neurotoxin-induced insults that lead to PD (Sun et al., 2017).

\section{AMPA Receptors}

AMPARs belong to the family of ionotropic glutamate receptors. They are thought to be the most significant mediators of excitatory neurotransmission in the CNS (Rogawski, 2011). They are assembled from four subunits (GluA1-4) and are mainly permeable to $\mathrm{Na}^{+}$and $\mathrm{K}^{+}$and to $\mathrm{Ca}^{2+}$ to a lesser extent. The subunit composition of AMPARs varies depending on the stage of development, region, and cell type (Song and Huganir, 2002). Phosphorylation of the GluA1 C-terminal tail regulates activity-dependent synaptic transport and channel features of the receptor (Esteban et al., 2003). Ser-831 and Ser845 are two phosphorylation sites of GluA1 that have been well-characterized. Phosphorylation at Ser-831 by CaMKII and protein kinase $\mathrm{C}$ (PKC) regulates channel conductance, whereas cyclic adenosine monophosphate (cAMP)/protein kinase A (PKA)-dependent phosphorylation at Ser-845 enhances channel open probability and promotes AMPAR internalization (Derkach 
et al., 1999). The PKA-dependent phosphorylation of GluA1 depends on the PKA scaffold AKAP150, which places PKA in proximity to its synaptic targets (Garcia-Alvarez et al., 2015) and cAMP-mediated dissociation of the regulatory subunit (rPKA) from the catalytic subunit (cPKA; Garcia-Alvarez et al., 2015). Both Ser-845 and Ser-831 phosphorylation sites play a role in LTP and LTD, forms of synaptic plasticity that are responsible for learning and memory (Esteban et al., 2003; Makino and Malinow, 2011).

Interestingly, STIM2 was shown to interact with AMPARs through a mechanism that is not associated with SOCE (GarciaAlvarez et al., 2015). Recent research showed that STIM2 induces the cAMP/PKA-dependent delivery of GluA1 to the PM (GarciaAlvarez et al., 2015). These authors suggested that STIM2 couples PKA to AMPARs and promotes the phosphorylation of GluA1 at Ser-845. They revealed a strong interaction between STIM2 and cPKA and weak STIM2 binding to rPKA and AKAP150, which may clarify the mechanism of interaction. Surprisingly, STIM2 and the phosphorylation of GluA1 at Ser-831 are negatively correlated. In STIM2-silenced neurons, phosphorylation at Ser831 is enhanced. These findings indicate that STIM2 regulates the phosphorylation of GluA1 at both Ser-845 and Ser-831 but in a different manner. In turn, STIM1 overexpression was shown to increase GluA1 phosphorylation at Ser-845 in hippocampal synaptoneurosomes (Majewski et al., 2017).

Our previous study found that STIM proteins in primary rat cortical neurons may also interact with AMPARs in a SOCE-dependent manner, meaning that when $\mathrm{ER} \mathrm{Ca}^{2+}$ stores are depleted, $\mathrm{Ca}^{2+}$ may enter through Orais, TRPC channels, and AMPARs (Gruszczynska-Biegala et al., 2016). The SOCE inhibitors ML-9 and SKF96365 decreased AMPA-induced Ca ${ }^{2+}$ influx, and the competitive AMPAR antagonists CNQX and NBQX inhibited SOCE. The induction of SOCE by thapsigargin resulted in AMPAR activation either directly through the recruitment of AMPARs to the $\mathrm{PM}$ or indirectly through unknown mechanisms. We also confirmed that both STIM1 and STIM2 proteins directly interacted with the GluA1 and GluA2 subunits of AMPARs. Moreover, STIM-AMPAR complexes appear to be located in ER-PM junctions (Gruszczynska-Biegala et al., 2016).

\section{NMDA Receptors}

NMDARs are ligand-gated ion channels that mediate $\mathrm{Ca}^{2+}$ influx when activated by glutamate, the main excitatory neurotransmitter in the mammalian CNS. NMDARs are tetramers that are composed of two glycine-binding NR1 (GluN1) and two glutamate-binding NR2 (GluN2) subunits (Cull-Candy and Leszkiewicz, 2004). Synaptic NMDARs consist mainly of NR1-NR2A or NR1-NR2A-NR2B receptors, and somatic NMDARs consist mainly of NR1-NR2B (Cull-Candy and Leszkiewicz, 2004). $\mathrm{Ca}^{2+}$ influx through NMDARs plays an important role in neuronal development, the formation of basal excitatory synaptic transmission, cell survival, and different forms of synaptic plasticity, such as LTP and LTD (Malenka and Bear, 2004; Nakazawa et al., 2004; Kerchner and Nicoll, 2008).
Pathologically high levels of glutamate and NMDA cause excitotoxicity by allowing high levels of $\mathrm{Ca}^{2+}$ to enter the cell (Sattler and Tymianski, 2000). Excessive NMDAR stimulation and prolonged increases in intracellular $\mathrm{Ca}^{2+}$ concentrations cause $\mathrm{Ca}^{2+}$ overload, which is considered a main cause of neuronal death in various neurodegenerative diseases that are associated with excitotoxicity, such as $\mathrm{HD}$ and $\mathrm{AD}$ (Marambaud et al., 2009; Pchitskaya et al., 2018; Serwach and Gruszczynska-Biegala, 2019). Attenuating intracellular $\mathrm{Ca}^{2+}$ overload is thus essential for limiting neuronal cell death under neuropathological conditions.

In hippocampal pyramidal neurons, SOCE can be activated by synaptic NMDAR stimulation, thus demonstrating its involvement in synaptic plasticity, such as LTP (Baba et al., 2003; Dittmer et al., 2017). Interestingly, glutamate release from neuronal terminals and NMDAR activation also induce SOCE in the PM of adjacent astrocytes possibly involving astrocytic mGluR5 (Lim et al., 2018). We recently reported that NMDARs contribute to $\mathrm{Ca}^{2+}$ influx in SOCE in rat cortical neurons (Gruszczynska-Biegala et al., 2020). The glutamate depolarization of neurons activates $\mathrm{Ca}^{2+}$ influx through NMDARs and Ltype VGCCs, releases $\mathrm{Ca}^{2+}$ from the ER (Simpson et al., 1995; Emptage et al., 1999, 2001; Dittmer et al., 2017), and aggregates and activates STIM1, which then triggers SOCE (Rae et al., 2000; Emptage et al., 2001; Dittmer et al., 2017). In recent work, we found that endogenous STIM1 and STIM2 interact in situ and in vitro and co-localize with endogenous NMDAR subunits in rat cortical neurons. Emptying $\mathrm{Ca}^{2+}$ from ER stores induces a decrease in the physical association between endogenous STIM1 and NR2B and between STIM2 and NR2A (Gruszczynska-Biegala et al., 2020). Additionally, STIMs were shown to modulate NMDA-evoked intracellular $\mathrm{Ca}^{2+}$ levels by interacting with them. These data suggest that STIM1 and STIM2 are negative regulators of NMDA-induced intracellular $\mathrm{Ca}^{2+}$ elevations in cortical neurons (Gruszczynska-Biegala et al., 2020), in which they have been shown to inhibit the activity of L-type VGCCs (Park et al., 2010; Wang et al., 2010; Dittmer et al., 2017).

\section{STIM INTERACTING PROTEINS}

Most of the aforementioned studies on STIM function in the CNS focused on its canonical function in $\mathrm{ER} \mathrm{Ca}^{2+}$ store refilling that results from the activation of SOCE. Recently, an increasing number of proteins have been reported to play a vital role in the regulation of Orai1-, TRPC-, and STIM1-associated $\mathrm{Ca}^{2+}$ signaling in both a SOCE-dependent and SOCE-independent manner in the CNS. This section focuses on several of the most important partners of STIM proteins that may also contribute to essential molecular processes in the CNS (Figure 3).

\section{mGluRs}

Group I metabotropic glutamate receptors (mGluRs) are widely distributed in the CNS and play a key role in synaptic transmission and plasticity. Excessive mGluR activation was shown in acute and chronic neurodegenerative disorders such as $\mathrm{PD}, \mathrm{AD}$, and $\mathrm{HD}$ (Crupi et al., 2019). The stimulation of 
group I mGluRs activates two signaling pathways (Hartmann et al., 2014). The first signaling pathway activates phospholipase $\mathrm{C}$ (PLC), thus inducing the formation of $\mathrm{IP}_{3}$, which interacts with $\mathrm{IP}_{3} \mathrm{Rs}$ to release $\mathrm{Ca}^{2+}$ from ER stores (Hartmann et al., 2014). The second signaling pathway involves the formation of slow excitatory post-synaptic potentials (EPSPs; Batchelor et al., 1994) and is mediated by TRPC3 (Hartmann et al., 2008).

STIM proteins also interact with TRPC3, and the STIMmGluR association has been widely investigated. Hartman et al. showed that following mGluR1 activation, STIM1 proteins oligomerize and evoke SOCE through TRPC3 channels (Hartmann et al., 2008, 2014). Consistent with this, Ng et al. found that the activation of group I mGluRs with 3,5-dihydroxyphenylglycine (DHPG) in hippocampal neurons stimulated STIM1 oligomerization and its transport to the PM (Ng et al., 2011). Another research group showed that STIM1 in cerebellar PNs participates in mGluR1-dependent synaptic transmission and thus regulates cerebellar motor behavior (Hartmann et al., 2014). In mice with the PN-specific deletion of STIM1, mGluR1-dependent signaling was eliminated, and both $\mathrm{IP}_{3}$-dependent $\mathrm{Ca}^{2+}$ release from the ER and TRPC3-mediated EPSCs were attenuated. The disruption of these two pathways abolished cerebellar motor behavior (Hartmann et al., 2014). The role of STIM1 in synaptic plasticity was also investigated in hippocampal slices from adult transgenic $\mathrm{Tg}$ (STIM1)Ibd mice. STIM1 overexpression appears to disrupt mGluR LTD that is induced by DHPG (Majewski et al., 2017).

A recent analysis by Tellios et al. showed that in the absence of neuronal nitric oxide synthase (nNOS)-derived NO signaling along with the higher expression of mGluR1, STIM1 expression and cluster density are elevated in PNs (Tellios et al., 2020). These authors suggested that the overactivation of mGluR1 results in $\mathrm{ER} \mathrm{Ca}^{2+}$ depletion and chronic STIM1 oligomerization. Because of the unlimited opening of TRPC 3 channels, $\mathrm{Ca}^{2+}$ entry through STIM1-gated TRPC3 channels may be elevated, further leading to a reduction of dendritic spine integrity in PNs (Tellios et al., 2020). In contrast, under physiological conditions, nNOS/NO signaling maintains $\mathrm{Ca}^{2+}$ homeostasis in neurons by reducing its influx through mGluR1 and inducing the $S$-nitrosylation of STIM1. As STIMs and Orais as well as NMDARs and mGluRs are expressed in the microvascular endothelial cells of the brain (LeMaistre et al., 2012; Negri et al., 2020), we can suspect that these endothelial receptors may also be regulated by STIMs. Especially since the $\mathrm{Ca}^{2+}$ response to glutamate by activating mGluR1 and mGluR5 is initiated by endogenous $\mathrm{Ca}^{2+}$ release from the ER through $\mathrm{IP}_{3} \mathrm{R}_{3}$ and sustained by SOCE, resulting in a rapid NO release (Negri et al., 2020).

\section{Homer Family Proteins}

Homer family proteins are post-synaptic scaffolding proteins that regulate glutamatergic signaling and intracellular $\mathrm{Ca}^{2+}$ concentrations in neurons (Chen et al., 2012). Homer was reported in neurological disorders, including $\mathrm{AD}$, TBI, and schizophrenia (Luo et al., 2012). These proteins are divided into two major groups: short-form Homerla proteins and long-form Homer1b/c, Homer2, and Homer3 proteins. Both groups have N-terminal EVH1 domain that is involved in protein interactions, and long-form proteins also have a C-terminal coiled-coil domain that is involved in self-association (Chen et al., 2012). Homer $1 \mathrm{~b} / \mathrm{c}$ has been shown to alter SOCE through an association with STIM1 and Orail in human platelets. This interaction between STIM1, Homer1b/c, and Orail is enhanced by thapsigargin (Jardin et al., 2012). Thapsigargin also induces Homer1, STIM1, and L-type VGCC associations in HEK-293 cells (Dionisio et al., 2015).

In the CNS, SOCE antagonists and STIM1-targeted smallinterfering RNA (siRNA) increase the expression of Homerla mRNA and the amount of Homerla protein (Li et al., 2013). The knockdown of Homerla expression partially reverses this effect. Moreover, SOCE inhibition appears to protect neurons against oxidative stress through the upregulation of Homerla expression (Li et al., 2013). SOCE inhibitors also prevented mitochondrial dysfunction and activation of mitochondrial apoptotic factors after $\mathrm{MPP}^{+}$injury. Since mitochondrial dysfunction is thought to play a crucial role in $\mathrm{PD}$, it seems that SOCE may be a potential target in the treatment of PD patients (Li et al., 2013).

Homerla affects STIM1-Orail associations, inhibits SOCE, and alleviates glutamate-induced cell death after oxidative stress injury (Rao et al., 2016). Both thapsigargin-induced and glutamate-mediated STIM1-Orail associations are attenuated by Homerla overexpression. After thapsigargin-induced ER Ca ${ }^{2+}$ store depletion, the association between STIM1 and Homerla decreases, whereas no change occurs in the Homerla-Orail association. These interactions between STIM1, Homerla, and Orail are similar to interactions between STIM1 and Homer1b/c or STIM1, Homer1b/c, and Orail (Jardin et al., 2012; Dionisio et al., 2015; Rao et al., 2016). Therefore, Homer1a might dissociate STIM1-Orail complexes and downregulate SOCE through negative competition with Homer1b/c (Rao et al., 2016). The regulation of Homerla and SOCE inhibition may be a potential therapeutic target for the treatment of neurological disorders, the etiology of which is associated with oxidative stress.

\section{SARAF}

SOCE-associated regulatory factor (SARAF) is a 339-aminoacid type I transmembrane protein that has exceptionally high transcript levels in neuronal tissues (Palty et al., 2012). It is assembled from a single $\mathrm{N}$-terminal transmembrane domain and a C-terminal domain that is located in the ER or PM (Palty et al., 2012; Albarran et al., 2016). The activation of SARAF involves the intraluminal region, whereas the interaction with SARAF engages the cytosolic region (Jha et al., 2013). Gene product of TMEM66, SARAF was identified as a biomarker linked to AD (Twine et al., 2011).

SARAF modulates STIM1-regulated $\mathrm{Ca}^{2+}$ entry, which includes both SOCE and $\mathrm{Ca}^{2+}$ influx through ARC channels (Palty et al., 2012; Albarran et al., 2016). SARAF prevents the spontaneous activation of STIM1, regulates STIM1-Orai1mediated SOCE, facilitates the slow $\mathrm{Ca}^{2+}$-dependent inactivation of SOCE, and promotes STIM1 deoligomerization after $\mathrm{Ca}^{2+}$ store refilling (Palty et al., 2012; Jha et al., 2013). SARAF was also reported to modulate cytosolic and $\mathrm{ER} \mathrm{Ca}^{2+}$ levels (Palty et al., 2012). The molecular mechanism of action of SARAF was described by Jha et al., who showed that SARAF interacts 
with the C-terminal inhibitory domain (CTID) of STIM1 to mediate the slow $\mathrm{Ca}^{2+}$-dependent inactivation (SCDI) of Orai1forming CRAC (Jha et al., 2013). Under resting conditions, when intracellular $\mathrm{Ca}^{2+}$ stores are filled with $\mathrm{Ca}^{2+}$, CTID facilitates the access of SARAF to the SOAR to keep STIM1 in an inactive state, resulting in the inhibition of STIM1-Orai communication. Upon $\mathrm{Ca}^{2+}$ store depletion, SARAF dissociates from STIM1 to allow the activation of STIM1-Orail complexes at ER-PM junctions, thereby leading to SOCE (Jha et al., 2013).

SARAF is constitutively expressed in the PM. It was also shown to modulate $\mathrm{Ca}^{2+}$ entry through ARC channels in the SH-SY5Y neuroblastoma cell line (Albarran et al., 2016). ARC channels are heteropentameric complexes that consist of three Orail and two Orai3 subunits and PM-resident STIM1. The overexpression of SARAF in neuroblastoma cells attenuated the arachidonic acid (AA)-induced $\mathrm{Ca}^{2+}$ response, and the transfection of SARAF siRNA enhanced AA-stimulated $\mathrm{Ca}^{2+}$ influx via ARC channels. The results suggest that SARAF is a negative regulator of AA-induced $\mathrm{Ca}^{2+}$ signaling (Albarran et al., 2016).

SARAF was also shown to interact with TRPC1 in a STIM1independent manner in both STIM1-defficient NG115-401L and endogenous STIM1-expressing SH-SY5Y neuroblastoma cells (Albarrán et al., 2016). The silencing of SARAF expression in STIM1-deficient cells increased TRPC1-mediated $\mathrm{Ca}^{2+}$ entry. In cells that endogenously expressed STIM1, the interaction between SARAF and TRPC1 was not associated with STIM1. This regulation of $\mathrm{Ca}^{2+}$ entry is thought to protect the cell from $\mathrm{Ca}^{2+}$ overload and adjust the influx of $\mathrm{Ca}^{2+}$, which was previously reported for the regulation of SOCE and ARC channels (Albarran et al., 2016). The silencing of SARAF expression did not influence TRPC6-mediated $\mathrm{Ca}^{2+}$ entry, in contrast to TRPC1, meaning that SARAF is unlikely to regulate the TRPC6 function. SARAF appears to play a negative regulatory role in both STIM1Orail- and STIM1-TRPC1-mediated $\mathrm{Ca}^{2+}$ entry by destabilizing STIM1/Orail complexes (Palty et al., 2012). Notably, a recent study reported a reduction of the expression of STIM1 and SARAF in the ischemic cortex, indicating that SARAF may be a new neuroprotective target for the treatment of stroke (La Russa et al., 2020).

\section{Septins}

Septins are a class of evolutionary conserved GTPases that are assembled into hexameric or octameric complexes organized into linear filaments or other higher-order structures. They function as diffusion barriers and intracellular scaffolds in cells during various cellular processes (Mostowy and Cossart, 2012). The altered septin function may contribute to synaptic dysfunction in neurodegenerative diseases (Marttinen et al., 2015).

Septins were shown to regulate SOCE in both non-excitable mammalian cells (Sharma et al., 2013) and Drosophila neurons (Deb and Hasan, 2016, 2019; Deb et al., 2016, 2020). In Drosophila, septins are classified into several groups: dSEPT1, dSEPT2, dSEPT4, dSEPT5 and dSEPT7. The dSEPT7, dSEPT1, and dSEPT4 groups form linear hexamers. dSEPT1 and dSEPT4 occupy the central position, and dSEPT7 is localized in terminal positions of the hexamer. These hexamers are then linked and form linear septin filaments (Mostowy and Cossart, 2012; Mavrakis et al., 2014). The molecular mechanism of SOCE regulation and contribution of different subgroups of septins to the regulation of SOCE is complex.

The simultaneous knockdown of dSEPT1 and dSEPT4 reduced SOCE in Drosophila flight circuit neurons (Deb et al., 2016). The knockdown of these subgroups results in the loss of septin filaments and loss of the diffusion barrier, which has a negative influence on Orai activation by STIM (Deb and Hasan, 2016). dSEPT1 and dSEPT4 appear to function as positive regulators of SOCE in Drosophila neurons (Deb et al., 2016; Deb and Hasan, 2019). On the other hand, reduction of dSEPT7 had no significant influence on SOCE in Drosophila neurons. Nevertheless, the reduction of dSEPT7 in primary neurons that had low levels of Drosophila STIM1 (dSTIM1) improved SOCE (Deb et al., 2016). Additionally, STIM1 is necessary for SOCE through Orai channels also in SEPT7 knockdown human neural progenitor cells (hNPCs; Deb et al., 2020). In resting neurons with low dSEPT7 expression, the intensity of dSTIM and resulting dSTIM-dOrai clusters that are observed near the ER-PM region increased (Deb et al., 2016, 2020). Similar STIM1 and Orail reorganization was shown at the cell surface in SEPT7 knockdown neurons that were differentiated from hNPCs (Deb et al., 2020). Deb et al. suggested that the partial reduction of dSEPT7 leaves hexameric complexes intact but results in the formation of smaller septin filaments because filament elongation requires dSEPT7 (Deb et al., 2016). Shorter SEPT7 filaments support dSTIM migration to the peripheral ER in resting neurons, promoting Orai channel opening independently from either $\mathrm{ER}-\mathrm{Ca}^{2+}$ store depletion or $\mathrm{Ca}^{2+}$ release through $\mathrm{IP}_{3}$ Rs. Similarly, SEPT4 regulates the number of the ER-PM junctions and enhances STIM1-Orail interactions within junctions in human cells (Katz et al., 2019). Store-independent dOrai $\mathrm{Ca}^{2+}$ influx results in higher cytosolic $\mathrm{Ca}^{2+}$ concentrations in resting neurons (Deb and Hasan, 2016). The loss of dSEPT7 influences the constitutive activation of Orai channels in resting neurons by uncoupling septin heteromers from ER-PM junctions, thus allowing the STIM interaction with Orai (Deb et al., 2016, 2020).

Septins (e.g., dSEPT1, dSEPT4, and SEPT7) appear to perform antagonistic rather than synergistic functions in Orai channel activation by STIM. This discrepancy may be caused by a different assembly of septins during complex formation and by differences in subsequent filament structure (Deb and Hasan, 2019). Altogether, these results could link alterations of septin expression to impairments in STIM1-dependent SOCE in human neurodegenerative diseases.

\section{Golli Proteins}

Golli proteins are isoforms of myelin basic protein (MBP) that are abundantly expressed in immune cells and the CNS (Paez et al., 2011). It is upregulated in adult OPCs and microglia in multiple sclerosis lesions (Filipovic et al., 2002). Myelin abnormalities have been implicated in neurodegenerative and neuropsychiatric diseases and Golli-MBP expression was increased in the aging brains (Siu et al., 2015). Golli proteins were shown to be components of remyelination that is caused by treatment with 
taxol in demyelinating transgenic mice, thus demonstrating their role in early stages of OPC proliferation and migration (Moscarello et al., 2002). Although no evidence suggests the occurrence of SOCE in oligodendroglia cells, this process was detected in OPCs and brain slices and shown to be mediated by STIM1 and TRPC1. Additionally, SOCE in these cells is positively modified by golli proteins that interact with STIM1 and TRPC1 (Paez et al., 2011). Changes in golli protein expression alter VGCCs and SOCs to mediate the migration and proliferation in OPCs that influence their maturation and survival ( $\mathrm{Paez}$ et al., 2009a,b; Paez et al., 2007). Another study showed that golli protein overexpression increases the mitogen-stimulated proliferation of OPCs through the activation of SOCE, which is essential for cell division (Paez et al., 2009a). In OPCs, the proliferation of golli protein-KO cells was less robust, and the duration of the cell cycle increased. Golli proteins were also reported to increase apoptotic cell death, which was associated with an increase in $\mathrm{Ca}^{2+}$ influx through VGCCs (Paez et al., 2009a). Notably, the C-terminus of STIM1 was also shown to bind to MBP in a brain extract in a pull-down assay, which is likely an epitope that is shared with golli protein (Walsh et al., 2010).

\section{POST and SERCA}

Several molecular mechanisms are responsible for the increase in cytosolic $\mathrm{Ca}^{2+}$ concentrations after cell depolarization, including SERCA, PMCA, NCX, and mitochondrial $\mathrm{Ca}^{2+}$ uptake. Among these mechanisms, SERCA and PMCA are regulated by STIM1, combined with an adaptor protein called partner of STIM1 (POST; Krapivinsky et al., 2011). After ER Ca ${ }^{2+}$ store depletion, the STIM1-POST complex binds to SERCA and keeps it close to $\mathrm{Ca}^{2+}$ entry sites on the $\mathrm{PM}$ to promote the refilling of ER $\mathrm{Ca}^{2+}$ stores (Krapivinsky et al., 2011). The STIM1-POST complex also inhibits PMCA activity, which is associated with $\mathrm{Ca}^{2+}$ outflow from the cytoplasm to the extracellular space (Ritchie et al., 2012). STIM1 appears to play opposing roles at the same time. However, SERCA contributes more to cytosolic $\mathrm{Ca}^{2+}$ clearance than PMCA, especially in PNs (Fierro et al., 1998). Thus, after PM depolarization, STIM1 reduces cytoplasmic $\mathrm{Ca}^{2+}$ concentrations. Additionally, Ryu et al. showed that STIM1 contributes to SERCA-dependent cytosolic $\mathrm{Ca}^{2+}$ clearance in the soma of firing PNs (Ryu et al., 2017). These authors proposed that the STIM1-POST complex may pull SERCA into the vicinity of VGCCs. If so, then SERCA could buffer $\mathrm{Ca}^{2+}$ influx more rapidly after depolarization, which can optimize the $\mathrm{Ca}^{2+}$-clearing and -buffering function of SERCA and prevent excessive cytosolic $\mathrm{Ca}^{2+}$ concentrations during repetitive firing (Ryu et al., 2017).

STIM1 mediates the sequestration of cytosolic $\mathrm{Ca}^{2+}$ ions by SERCA. It may also regulate neuronal excitability. Nevertheless, still unknown is whether this occurs only with high-firing neurons, such as PNs, or also with slow-firing neurons, such as pyramidal and cortical neurons (Ryu et al., 2017).

In this context it is worth noting that the function of SERCA can be also regulated by ER lumen residents calreticulin and ERp57 oxidoreductase (Li and Camacho, 2004). Thus, we suspect that these proteins may interact with STIM in neurons, especially since they are expressed therein (Coe and
Michalak, 2010). ERp57 and STIM1 formed complexes in vivo and in vitro to inhibit STIM oligomerization and SOCE in mouse embryonic fibroblasts (Prins et al., 2011). Interestingly, in megakaryocytes from healthy individuals, calreticulin regulated SOCE activation through interaction with ERp57 and STIM1. In megakaryocytes from patients with mutated calreticulin, destabilization of the complex between calreticulin, ERp57 and STIM1 was observed, leading to enhanced SOCE and thus to abnormal cell proliferation (Di Buduo et al., 2020). ERp57 is associated not only with myeloproliferative neoplasms, but also with many disease states of CNS, such as prion disorders and AD, where ERp57 and calreticulin have been shown to prevent amyloid aggregation (Coe and Michalak, 2010). Similarly, SERCA-mediated $\mathrm{Ca}^{2+}$ dysregulation is associated with neuropathological conditions, such as affective disorders and neurogenerative diseases (Britzolaki et al., 2020).

\section{$\mathrm{IP}_{3}$ Rs}

Receptors that activate PLC cause the formation of $\mathrm{IP}_{3}$, which triggers both $\mathrm{Ca}^{2+}$ release from ER stores through $\mathrm{IP}_{3} \mathrm{Rs}$ and $\mathrm{Ca}^{2+}$ influx from the extracellular milieu, which is mediated by SOCE. $\mathrm{IP}_{3}$ Rs are thought to regulate SOCE by mediating ER $\mathrm{Ca}^{2+}$ release. Under physiological conditions, store depletion causes STIM and $\mathrm{IP}_{3} \mathrm{R}$ accumulation near the PM, an association between STIM and Orai, and the activation of SOCE. In Drosophila neurons with mutant $\mathrm{IP}_{3} \mathrm{Rs}$, SOCE was attenuated (Chakraborty et al., 2016; Deb et al., 2016) and this attenuation was reversed by STIM and Orai overexpression. The authors speculated that after $\mathrm{ER} \mathrm{Ca}^{2+}$ store depletion in Drosophila neurons, $\mathrm{IP}_{3} \mathrm{R}$ translocation to the ER-PM junction triggers the coupling of STIM to Orai, leading to the activation of SOCE (Chakraborty et al., 2016).

The same research group also reported the enhancement of spontaneous $\mathrm{Ca}^{2+}$ influx from the extracellular milieu and loss of SOCE in Drosophila pupal neurons with mutant $\mathrm{IP}_{3} \mathrm{Rs}$ (Chakraborty and Hasan, 2017). Both spontaneous $\mathrm{Ca}^{2+}$ influx and the attenuation of SOCE were reversed by dOrai and dSTIM overexpression. Additionally, the expression of VGCCs decreased, and the expression of trp mRNAs and TRPC protein increased in mutant neurons, suggesting that these channels might be associated with the increase in spontaneous $\mathrm{Ca}^{2+}$ influx. Spontaneous $\mathrm{Ca}^{2+}$ influx likely compensates for the loss of SOCE in Drosophila $\mathrm{IP}_{3} \mathrm{R}$ mutant neurons and maintains intercellular $\mathrm{Ca}^{2+}$ homeostasis (Chakraborty and Hasan, 2017). The overexpression of dSTIM in insulin-producing neurons and aminergic neurons also improves SOCE and restores flight in a flightless Drosophila $\mathrm{IP}_{3} \mathrm{R}$ mutant (Agrawal et al., 2010). These authors suggested that $\mathrm{IP}_{3} \mathrm{R}$-mediated $\mathrm{Ca}^{2+}$ release couples to SOCE via dSTIM/dOrai in Drosophila flight circuit neurons, thereby allowing dSTIM to compensate for impairments in $\mathrm{IP}_{3} \mathrm{R}$ function (Agrawal et al., 2010).

No evidence has been reported that the contribution of $\mathrm{IP}_{3} \mathrm{R}$ to SOCE in Drosophila occurs in mammalian neurons. However, in some mammalian cells, $\mathrm{IP}_{3} \mathrm{Rs}$ have been shown to co-localize with Orail (Lur et al., 2011) and interact with STIM1, Orai1, and TRPCs (Hong et al., 2011). The expression of $\mathrm{IP}_{3} \mathrm{R}$ isoform $\left(\mathrm{IP}_{3} \mathrm{R}_{3}\right)$ was shown to be significantly lower in STIM1-deficient 
SH-SY5Y cells, meaning that STIM1 is a positive regulator of ITPR3 gene expression in these cells (Pascual-Caro et al., 2020). $\mathrm{IP}_{3} \mathrm{R}_{3}$ is a $\mathrm{Ca}^{2+}$ channel that is localized mainly at the ERmitochondrion junction, which transfers $\mathrm{Ca}^{2+}$ from the ER to mitochondria (Ivanova et al., 2014). Thus, STIM1 deficiency leads to a decrease in mitochondrial $\mathrm{Ca}^{2+}$ concentrations, leading to cell death. The overexpression of $\mathrm{IP}_{3} \mathrm{R}_{3}$ restores mitochondrial $\mathrm{Ca}^{2+}$ homeostasis and bioenergetics, ATP production, and cell survival in STIM1-KO neuronal-like cells (Pascual-Caro et al., 2020). These results provide evidence of a novel STIM1-IP $\mathrm{I}_{3}$-mediated pathway of mitochondrial $\mathrm{Ca}^{2+}$ levels, the dysregulation of which contributes to neurodegeneration. Mitochondria from AD patients have lower $\mathrm{Ca}^{2+}$ uptake (Kumar et al., 1994), which is attributed to lower $\mathrm{IP}_{3} \mathrm{R}_{3}$ and STIM1 levels (Pascual-Caro et al., 2020).

\section{EB1 and EB3}

The dynamic structure of dendritic spines is preserved mainly by actin filaments, and microtubules (MTs) are cytoskeletonorganizing components localized in dendrites and axons (Majewski and Kuznicki, 2015; Wu et al., 2017). Microtubules have been shown to enter dendritic spines and trigger spine head enlargement ( $\mathrm{Gu}$ et al., 2008; Hu et al., 2008). This transport of MTs into dendritic spines appears to be involved in mechanisms of synaptic plasticity. Microtubule plus-ends contain end-binding $(\mathrm{EB})$ proteins, which are divided into three types: EB1, EB2, and EB3 and have been shown to interact with STIM1 (Akhmanova and Steinmetz, 2010).

EB1/EB3-STIM1 complexes mediate ER movement in nonexcitable cells (Grigoriev et al., 2008; Honnappa et al., 2009; Asanov et al., 2013). The STIM1-EB association sequesters STIM1 in MTs and prevents the excessive activation of SOCE (Chang et al., 2018). STIM1 regulates the dynamics of EB1/EB3, coupling the ER to MTs within filopodia and thus controlling growth cones in the nascent nervous system (Pavez et al., 2019). Additionally, recent research demonstrated that EB3 forms complexes with STIM2 that promote the formation of mushroom spines in hippocampal neurons, and the disintegration of these complexes results in the loss of mushroom spines (Pchitskaya et al., 2017). The overexpression of EB3 increases the proportion of mushroom spines and rescues their deficiency in hippocampal neurons in an $\mathrm{AD}$ mouse model. EB3 overexpression also rescues the loss of mushroom spines after STIM2 knockdown, whereas STIM2 overexpression does not restore mushroom spines after EB3 depletion. Neither STIM2 overexpression nor the activation of hippocampal TRPC6 increases spine neuronal SOCs or the proportion of mushroom spines in WT neurons. EB3 recruits various proteins to dendritic spines during synaptic plasticity, and STIM2 may be one of these cargo proteins (Pchitskaya et al., 2017). EB3 is involved in the regulation of dendritic spine morphology partly through its association with STIM2. Therefore, targeting EB3-STIM2 complexes may stabilize dendritic spines in AD patients (Pchitskaya et al., 2017).

\section{Synaptopodin}

In cultured neurons, STIM1 interacts with anchoring proteins in the dendritic spine apparatus that consists of laminar smooth
ER stacks. Synaptopodin (SP) is localized between ER stacks of the spine apparatus. This cytosolic actin and $\alpha$-actinin-binding protein has been shown to be essential for the formation of this organelle (Deller et al., 2003). Synaptopodin is more common in spines with large-volume spine heads, where it regulates synaptic plasticity by controlling spine head enlargement during LTP in the CA1 region of the hippocampus and enhances glutamateinduced $\mathrm{Ca}^{2+}$ release in dendritic spines of cultured hippocampal neurons (Deller et al., 2003; Vlachos et al., 2009; Korkotian et al., 2014). Synaptopodin deficiency alleviated the AD symptoms in the $3 \mathrm{xTg}$ mice and restores normal synaptic plasticity (Aloni et al., 2019).

Synaptopodin was recently shown to regulate activitydependent $\mathrm{Ca}^{2+}$ signaling by recruiting STIM1 to the postsynaptic density (PSD; Korkotian et al., 2014; Segal and Korkotian, 2014). In primary hippocampal neurons, SP colocalizes with STIM1 (Korkotian et al., 2014). The localization of STIM1 in spines depended on SP, in which this protein preferentially located STIM1 to mushroom spines, where this association was especially evident (Korkotian et al., 2014). These results indicate that SP interacts with STIM and Orai and thus may regulate the functionality of $\mathrm{Ca}^{2+}$ stores and determine synaptic plasticity.

As SP belongs to actin-binding proteins, it would be interesting to investigate whether STIM could also directly interact with actin in dendritic spines. It is worth mentioning the regulatory role of actin in spine morphogenesis and stabilization that is necessary for memory formation, the role in mechanisms related to synaptic plasticity and the contribution to $\mathrm{AD}$ pathology (Basu and Lamprecht, 2018; Pelucchi et al., 2020). Previous research has demonstrated that STIM1 may interact with actin, and actin remodeling was required to move STIM to the PM after store depletion in human platelets. Furthermore, the polymerization of actin filaments was necessary for association of STIM1 with TRPC1 (López et al., 2006). Similarly, actin fibers were shown to be involved in an alternatively spliced long variant of STIM1 oligomerization that precedes activation of Orail in myoblasts (Darbellay et al., 2011). Notably, Trebak's group reported that STIM1 controls formation of actin stress fibers, independently of Orail and $\mathrm{Ca}^{2+}$, thus thrombin-mediated disruption of endothelial barrier function (Shinde et al., 2013).

\section{Presenilins and CaMKII}

Familial Alzheimer's disease (FAD) is caused by a dominant inherited mutation of presenilins (PSs; PS1 and PS2) and amyloid- $\beta$ (A $\beta$ ) precursor protein (APP; Chakroborty and Stutzmann, 2014). Presenilins constitute catalytic components of the $\gamma$-secretase complex, which cleaves transmembrane APP to produce A $\beta$. PS1 mutations have been shown to change APP cleavage in favor of producing $A \beta$. This peptide accumulates, causing neuronal death in the cerebral cortex and hippocampal neurons, contributing to cognitive impairment and other pathological hallmarks of AD (Chakroborty and Stutzmann, 2014).

Endogenous PS1 and STIM1 have been shown to interact in human SH-SY5Y neuroblastoma cells and mouse primary cortical neurons (Tong et al., 2016). Tong et al. defined 
STIM1 as a new substrate of $\gamma$-secretase in a PS model of AD. The PS1-accociated $\gamma$-secretase complex cleaves the STIM1 transmembrane domain, reducing ORAI activation and diminishing SOCE (Tong et al., 2016). Dendritic spines in hippocampal neurons with mutant PS1 are destabilized, which is reversed by both a $\gamma$-secretase inhibitor and STIM1 overexpression. Although, the cleavage of STIM2 has not yet been established, its structural similarity to STIM1 suggests that it may also be a target of $\gamma$-secretase. Ryazantseva et al. reported the enhancement of SOCE in hippocampal neurons with a PS1 $\triangle$ E9 mutation (Ryazantseva et al., 2018). This PS1 mutation excludes 28 amino acids from the proteolytic cleavage site, resulting in the accumulation of uncleaved proteins. STIM1 accumulation results in its enhanced relocation to the PM, increasing the Orai1-TRPC association and enhancing SOCE (Ryazantseva et al., 2018). In turn, SOCE was attenuated directly in neurons from transgenic mice expressing human mutant PS1 A246E (Herms et al., 2003). The effect of PS1 on STIM1 appears to differ depending on the type of mutation. Greotti et al. reported that both PS1 and PS2 in SH-SY5Y cells reduce SOCE by reducing STIM1 expression levels (Greotti et al., 2019). However, this reduction does not depend on $\gamma$-secretase activity. Lower amounts of STIM1 protein were also found in FAD PS-expressing cells that were treated with a $\gamma$-secretase inhibitor. Moreover, chronic ER $\mathrm{Ca}^{2+}$ depletion or alterations of STIM1 expression levels did not affect SOCE under resting conditions (Greotti et al., 2019). These results may be considered an adaptive consequence of a prolonged reduction of $\mathrm{ER} \mathrm{Ca}^{2+}$ levels.

Interestingly, $\mathrm{A} \beta$ itself decreases both STIM1 and STIM2 expression. Several studies have reported a link between A $\beta$ mediated STIM2 downregulation and the loss of synapses in animal models of AD (Bojarski et al., 2009; Fonseca et al., 2015; Popugaeva et al., 2015; Sanati et al., 2019). STIM2 protects mushroom spines from toxic effects of amyloid oligomers in vitro and in vivo in models of amyloid synaptotoxicity (Popugaeva et al., 2015). Sanati et al. reported that gold nanoparticles (AuNPs) reversed deteriorations of memory and spatial learning in $A \beta$-treated rat hippocampal neurons (Sanati et al., 2019). AuNPs delay the elongation of $A \beta$ and dissociate existing $A \beta$ to less toxic form, enhanced the expression of STIM protein, and potentiated the cAMP/PKA signaling cascade that modulates synaptic plasticity and influences learning and memory (Waltereit and Weller, 2003; Sanati et al., 2019). Additionally, AuNPs may directly increase cAMP levels and consequently recruit STIM2 and PKA to potentiate GluR1-dependent synaptic plasticity (Sanati et al., 2019).

In PS1 KI neurons, STIM2-mediated SOCE activates CaMKII and thus stabilizes mushroom spines (Sun et al., 2014). STIM2 is abundantly expressed in dendritic spines of hippocampal neurons, where it co-localizes with CaMKII. STIM2 overexpression rescues SOCE, restores CaMKII activity, and prevents dendritic spine loss. The conditional deletion of STIM2 reduced synaptic SOCE, thereby causing the loss of mushroom spines and eventually leading to the death of hippocampal neurons in mice that expressed FAD-associated PS1 variants (Sun et al., 2014). On the other hand, STIM2 was reported to inhibit $\mathrm{I}_{\mathrm{CRAC}}$ and SOCE amplitude and enhance intracellular $\mathrm{Ca}^{2+}$ stores through PS1 M146V mutant expression in a cellular model of AD (Ryazantseva et al., 2013). Mushroom spine loss also occurred in an APP-KI mouse model of AD, which was reported to be attributable to the accumulation of $\mathrm{A} \beta$ in the medium of APP-KI neurons (Zhang et al., 2015). A $\beta$ overactivates mGluR5, leading to higher $\mathrm{ER} \mathrm{Ca}^{2+}$ levels, the downregulation of STIM2 expression, impairments in synaptic SOCE, and lower CaMKII activity (Sun et al., 2014; Popugaeva et al., 2015; Zhang et al., 2015). The pharmacological inhibition of mGluR5 or overexpression of STIM2 restores synaptic SOCE and prevents mushroom spine loss. Downregulation of the synaptic STIM2-SOCE-CaMKII pathway causes the loss of mushroom spines in both PS1-KI and APP-KI models of AD. Moreover, TRPC6 and Orai2 have been shown to form complexes with STIM2 in hippocampal dendritic spines (Zhang et al., 2016). Thus, TRPC6 activation, STIM2 overexpression, and SOCE positive modulators can rescue mushroom spine loss in hippocampal neurons from both PS-KI and APP-KI mouse models of AD (Sun et al., 2014; Zhang et al., 2016).

\section{OTHER STIM-INTERACTING MOLECULES}

Recent studies have shown other regulators of STIM proteins, such as transcription factors and proteasome inhibitors that may negatively modulate their function. Here, we describe three such regulators.

\section{NEUROD2}

Neurogenic differentiation factor 2 (NEUROD2) is one of the most important neurogenic transcription factors in the CNS (Guner et al., 2017), which mutation is associated with schizophrenia (Dennis et al., 2019). Contrary to previous research that showed that NEUROD2 is a transcriptional activator (Fong et al., 2012; Bayam et al., 2015), a recent study suggested that it may also limit Stim1 expression in cortical neurons and consequently regulate $\mathrm{Ca}^{2+}$ influx in SOCE (Guner et al., 2017). Using a chromatin immunoprecipitation and sequencing approach in mouse postnatal cerebral cortical tissue, NEUROD2 was found to bind to an intronic element within the Stim1 gene. The knockdown of Neurod2 expression in cortical neurons increased STIM1 protein expression and resulted in the upregulation of SOCE, whereas its overexpression decreased SOCE. NEUROD2 activity is induced by $\mathrm{Ca}^{2+}$ influx via VGCCs, and depolarization-mediated $\mathrm{Ca}^{2+}$ influx via VGCCs appears to activate NEUROD2, which in turn fine-tunes the expression of STIM1 and SOCE and results in the STIM1-dependent inhibition of L-type VGCCs (Guner et al., 2017).

\section{Sp4}

$\mathrm{Sp} 4$ is a transcription factor that regulates neuronal morphogenesis and function. Its stability depends on membrane potential. Sp4 level was increased in the brains of $\mathrm{AD}$ patients and reduced in the brains of bipolar disorder patients (Boutillier et al., 2007; Pinacho et al., 2011). A recent study reported that the maximal activation of SOCE under resting conditions promotes the degradation of Sp4 in cerebellar granule neurons 
in vitro (Lalonde et al., 2014). The lowering of extracellular $\mathrm{K}^{+}$levels reduces neuronal excitability and stimulates the depletion of $\mathrm{ER} \mathrm{Ca}^{2+}$ stores, resulting in STIM1 migration to the ER-PM junction and SOCE activation. SOCE inhibitors prevent the ubiquitination and degradation of $\mathrm{Sp} 4$ during low extracellular $\mathrm{K}^{+}$levels. STIM1 knockdown also inhibits the degradation of Sp4, whereas a constitutively active STIM1 mutant (STIM1D76A) decreased Sp4 protein levels after depolarization (Lalonde et al., 2014). Neurons that were transfected with STIM1D76A were less likely to show immunopositive nuclei with Sp4 than WT STIM1. These findings suggest that STIM1 regulates Sp4 protein, meaning that $\mathrm{Sp} 4$ is a downstream effector of STIM1 and STIM1-mediated SOCE. We can assume that dysregulation of STIM1-mediated SOCE may induce abnormal Sp4 expression and promote the development of disorders such as bipolar disorder or AD.

\section{Proteasome Inhibitors}

A recent study showed that sub-lethal doses of proteasome inhibitors, such as MG-132 and clasto-lactacystin- $\beta$-lactone (LA), decreased STIM1 and STIM2 levels in primary rat cortical neurons but did not affect either Orail or TRPC1 (Kuang et al., 2016). The loss of STIM1 and STIM2 proteins was also observed in SH-SY5Y neuroblastoma cells that had low levels of the proteasome subunit $\beta$ type 5. Additionally, MG-132 and LA promoted autophagy and STIM1/STIM2 mobilization to lysosomes. Thus, inhibition of the ubiquitin-proteasome system, common in neurodegenerative disorders, may disrupt $\mathrm{Ca}^{2+}$ homeostasis by suppressing SOCE.

\section{CONCLUDING REMARKS}

Calcium homeostasis in the CNS is vital for cell maintenance. As a second messenger, $\mathrm{Ca}^{2+}$ participates in plenty physiological processes and its level is regulated in a comprehensive way via the components localized in the PM (ion channels, exchangers, and pumps), as well as the components localized in the mitochondria, ER, Golgi apparatus, and nucleus. Under pathological conditions, $\mathrm{Ca}^{2+}$ homeostasis is dysregulated, with increased cytoplasm, mitochondrial, and changed $\mathrm{ER} \mathrm{Ca}^{2+}$ concentration leading to apoptosis (Ureshino et al., 2019). Since the increasing evidence of the relevance of $\mathrm{Ca}^{2+}$ homeostasis in neuroprotection, we focused on the expression and function of $\mathrm{Ca}^{2+}$ signaling-related proteins, STIM partners and effectors, in terms of the effects on $\mathrm{Ca}^{2+}$ regulation and its potential use in the alleviation of the symptoms of neurodegenerative diseases.

Since the discovery of STIM and Orai proteins 15 years ago, they have been found to be the main components of SOCE but not the only components. Experimental evidence that was reviewed herein clearly demonstrates that STIM-Oraimediated SOCE in the CNS is influenced by several regulators and STIMs have several effectors. We summarized the existing knowledge of target molecules of STIM proteins (Table 1 and Figure 3).

We can distinguish both positive (SEPT1, SEPT4, golli proteins, SP, POST, and EB) and negative (Homer, SARAF,
SEPT7, PS1, NEUROD2, and proteasome inhibitors) regulators of STIM that affect STIM expression and structure and its movement to the PM or activation of Orai channels (Table 1). The majority of these regulators have an impact on STIMOrai interactions, but both golli proteins and SARAF also influence STIM-TRPC associations. Interestingly, some of the regulators appear to function in two ways. SEPT1 and SEPT4 increase STIM-Orai-dependent SOCE, whereas SEPT7 inhibits STIM migration to ER-PM junctions and Orai activation. In turn, EB3 can restore the loss of mushroom spines after knocking down STIM2 (i.e., a regulator of STIM). Conversely, the dynamics of EB1/EB3 are regulated by STIM1 (i.e., an effector of STIM). Notably, in contrast to golli proteins in immune cells where it negatively regulates STIM-dependent SOCE, golli proteins in the brain appear to have a positive regulatory action on SOCE activity that is mediated by STIM1. We assume that the action of golli proteins differs depending on the type of cell tested. However, the exact molecular mechanisms that underlie the STIM1-golli protein interaction have not yet been defined.

The main effectors of STIM proteins are Orai and TRPC, which together constitute molecular components of SOCE. The evidence gathered in this review suggests that disturbances of the STIM-Orai- and STIM-TRPC-mediated SOCE pathway contribute to the pathogenesis of diverse neurodegenerative diseases. The STIM-Orai association is also vital for the regulation of neurogenesis in mammalian cells and production of proinflammatory cytokines in astroglia and murine microglia. In turn, the STIM-TRPC interaction is essential for the survival of DNs in animal models of PD. In addition to Orai and TRPC, STIM proteins can also control $\mathrm{Ca}^{2+}$ influx via other molecular channels, including L-type VGCCs, and receptors, such as AMPARs, NMDARs, mGluR1, and mGluR5. The interaction between STIM proteins and their molecular targets can both increase and decrease $\mathrm{Ca}^{2+}$ influx from the extracellular milieu to the cytoplasm. Associations between STIMs and Orai, TRPC, AMPARs, mGluR1, and mGluR5 elevate $\mathrm{Ca}^{2+}$ influx, whereas $\mathrm{Ca}^{2+}$ influx via L-type VGCCs and NMDARs is inhibited by STIMs. Interestingly, maximal SOCE activation occurs under resting conditions, whereas VGCC and NMDAR activation requires cell membrane depolarization. Depolarization activates $\mathrm{Ca}^{2+}$ influx via both NMDARs and L-type VGCCs and decreases $\mathrm{Ca}^{2+}$ content in the ER, thereby enhancing STIM1-mediated SOCE and decreasing $\mathrm{Ca}^{2+}$ influx via VGCCs and NMDARs. Thus, STIM proteins in neurons may regulate $\mathrm{Ca}^{2+}$ influx under both resting and action potential.

The relationship between STIM proteins and glutamate receptors is essential for different forms of synaptic plasticity. In primary rat hippocampal neurons, STIM2 promotes the phosphorylation and surface delivery of AMPARs, contributing to LTP. SOCE can be activated by synaptic NMDARs, thus also influencing LTP. Additionally, STIM1 was shown to control the plasticity of L-type VGCC-dependent dendritic spines in PNs and strengthen mGluR1-dependent synaptic transmission, thereby regulating cerebellar motor behavior. Other STIM 
TABLE 1 | STIM target molecules.

\begin{tabular}{|c|c|c|c|c|}
\hline $\begin{array}{l}\text { Interacting } \\
\text { protein }\end{array}$ & $\begin{array}{l}\text { Identity of binding } \\
\text { protein }\end{array}$ & Subcellular location & Function & STIM isoform \\
\hline Orai & $\mathrm{Ca}^{2+}$ channel & PM & Positive effector & STIM1 STIM2 \\
\hline VGCC & $\mathrm{Ca}^{2+}$ channel & PM & Negative effector & STIM1 \\
\hline AMPAR & Ionotropic receptor & PM & Positive effector & STIM1 STIM2 \\
\hline septin & GTPase & PM lipids (cytoskeleton) & Positive/negative regulator & dSTIM \\
\hline Homer & Scaffolding protein & PSD (cytoskeleton) & Negative regulator & STIM1 \\
\hline SP & Actin-binding protein & PSD (cytoskeleton) & Positive regulator & STIM1 \\
\hline CaMKII & Kinase & PSD (cytoskeleton) & Positive effector & STIM2 \\
\hline POST & $\begin{array}{l}\text { Transmembrane } \\
\text { protein }\end{array}$ & ER (predominant), PM & Positive regulator & STIM1 \\
\hline SERCA & $\mathrm{Ca}^{2+}$-ATPase & ER & Positive effector & STIM1 \\
\hline $\mathrm{IP}_{3} \mathrm{R}$ & $\mathrm{Ca}^{2+}$ channel & ER & $\begin{array}{l}\text { Positive } \\
\text { regulator/effector }\end{array}$ & dSTIM STIM1 \\
\hline PS1 & $\begin{array}{l}\mathrm{Ca}^{2+} \text {-leak channel, } \\
\text { endoprotease }\end{array}$ & ER & Negative regulator & STIM1 STIM2 \\
\hline NEUROD2 & Transcription factor & Nucleus & Negative regulator & STIM1 \\
\hline Sp4 & Transcription factor & Nucleus & Negative effector & STIM1 \\
\hline MG-132/LA & $\begin{array}{l}\text { Proteasome } \\
\text { inhibitors }\end{array}$ & $\begin{array}{l}\text { Cytoplasm/ } \\
\text { nucleus }\end{array}$ & Negative regulator & STIM1 STIM2 \\
\hline
\end{tabular}

PM, plasma membrane; $E R$, endoplasmic reticulum; $P S D$, post-synaptic density.

protein effectors and regulators that reside outside the PM also contribute to synaptic plasticity. Synaptopodin recruits STIM1 to the PSD and regulates the function of $\mathrm{Ca}^{2+}$ stores and plasticity of spine heads during LTP. After cell depolarization, the STIM1-POST complex binds to SERCA and keeps it in close proximity to L-type VGCCs to promote ER $\mathrm{Ca}^{2+}$ replenishment during repetitive firing, regulating neuronal excitability and plasticity. The STIM1-POST complex appears to prevent excessive $\mathrm{Ca}^{2+}$ concentrations in the cytoplasm. In turn, the STIM1-IP $\mathrm{R}_{3}$ interaction regulates basal $\mathrm{Ca}^{2+}$ levels in mitochondria. Interestingly, the dysregulation of both cytosolic and mitochondrial $\mathrm{Ca}^{2+}$ levels contributes to neurodegeneration in AD. In turn, the STIM2-EB3 and STIM2-CaMKII complexes promote the formation of mushroom spines and stabilize them. Targeting these complexes could be a novel way of stabilizing dendritic spines and thus improve memory in AD patients.

Studying STIM proteins and their partners in different subcellular compartments enables us to understand a wide range of processes that are regulated by these proteins.
Future studies should examine the ways in which these regulators act in concert to modulate STIM activity during $\mathrm{Ca}^{2+}$ influx into the cell. The precise molecular mechanisms of action of STIMs together with these all regulators (e.g., in the activation/inactivation of Orai channels) also remain to be explored. Such studies will help to elucidate the pathological mechanisms that are involved in the development of various neurodegenerative diseases (e.g., AD, PD, and HD), affective disorders (schizophrenia, bipolar disorder), chronic pain, oxidative trauma, brain trauma, stroke, and epilepsy. Therefore, it appears that STIM proteins and their modulators/effectors may have potential therapeutic applications for the treatment of these diseases.

\section{AUTHOR CONTRIBUTIONS}

KS wrote and commented on the manuscript and prepared the figures. JG-B designed the manuscript, contributed to writing it, and prepared the final version. All authors contributed to the article and approved the submitted version. 


\section{FUNDING}

This study was supported by funds from the National Science Centre (research project no. 2017/26/E/NZ3/01144 to JG-B).

\section{REFERENCES}

Agrawal, N., Venkiteswaran, G., Sadaf, S., Padmanabhan, N., Banerjee, S., and Hasan, G. (2010). Inositol 1,4,5-trisphosphate receptor and dSTIM function in Drosophila insulin-producing neurons regulates systemic intracellular calcium homeostasis and flight. J. Neurosci. 30, 1301-1313. doi: 10.1523/JNEUROSCI.3668-09.2010

Ahsan, A., Zheng, Y. R., Wu, X. L., Tang, W. D., Liu, M. R., Ma, S. J., et al. (2019). Urolithin A-activated autophagy but not mitophagy protects against ischemic neuronal injury by inhibiting ER stress in vitro and in vivo. CNS Neurosci. Ther. 25, 976-986. doi: 10.1111/cns.13136

Akhmanova, A., and Steinmetz, M. O. (2010). Microtubule +TIPs at a glance. J. Cell Sci. 123, 3415-3419. doi: 10.1242/jcs.062414

Albarrán, L., López, J. J., Gómez, L. J., Salido, G. M., and Rosado, J. A. (2016). SARAF modulates TRPC1, but not TRPC6, channel function in a STIM1-independent manner. Biochem. J. 473, 3581-3595. doi: 10.1042/BCJ201 60348

Albarran, L., Lopez, J. J., Woodard, G. E., Salido, G. M., and Rosado, J. A. (2016). Store-operated $\mathrm{Ca}^{2+}$ entry-associated regulatory factor (SARAF) plays an important role in the regulation of arachidonate-regulated $\mathrm{Ca}^{2+}$ (ARC) channels. J Biol Chem. 291, 6982-6988. doi: 10.1074/jbc.M115.704940

Aloni, E., Oni-Biton, E., Tsoory, M., Moallem, D. H., and Segal, M. (2019). Synaptopodin deficiency ameliorates symptoms in the $3 \times \mathrm{Tg}$ mouse model of Alzheimer's disease. J. Neurosci 39, 3983-3992. doi: 10.1523/JNEUROSCI.2920-18.2019

Ambudkar, I. S., de Souza, L. B., and Ong, H. L. (2017). TRPC1, Orail, and STIM1 in SOCE: friends in tight spaces. Cell Calcium 63, 33-39. doi: 10.1016/j.ceca.2016.12.009

Asanov, A., Sherry, R., Sampieri, A., and Vaca, L. (2013). A relay mechanism between EB1 and APC facilitate STIM1 puncta assembly at endoplasmic reticulum-plasma membrane junctions. Cell Calcium 54, 246-256. doi: 10.1016/j.ceca.2013.06.008

Baba, A., Yasui, T., Fujisawa, S., Yamada, R. X., Yamada, M. K., Nishiyama, N., et al. (2003). Activity-evoked capacitative $\mathrm{Ca}^{2+}$ entry: implications in synaptic plasticity. J. Neurosci. 23, 7737-7741. doi: 10.1523/JNEUROSCI.23-21-07737.2003

Basu, S., and Lamprecht, R. (2018). The role of actin cytoskeleton in dendritic spines in the maintenance of long-term memory. Front. Mol. Neurosci. 11, 143. doi: $10.3389 /$ fnmol.2018.00143

Batchelor, A. M., Madge, D. J., and Garthwaite, J. (1994). Synaptic activation of metabotropic glutamate receptors in the parallel fibrePurkinje cell pathway in rat cerebellar slices. Neuroscience 63, 911-915. doi: 10.1016/0306-4522(94)90558-4

Bayam, E., Sahin, G. S., Guzelsoy, G., Guner, G., Kabakcioglu, A., and Ince-Dunn, G. (2015). Genome-wide target analysis of NEUROD2 provides new insights into regulation of cortical projection neuron migration and differentiation. BMC Genomics 16:681. doi: 10.1186/s12864-015-1882-9

Berna-Erro, A., Braun, A., Kraft, R., Kleinschnitz, C., Schuhmann, M. K., Stegner, D., et al. (2009). STIM2 regulates capacitive $\mathrm{Ca}^{2+}$ entry in neurons and plays a key role in hypoxic neuronal cell death. Sci Signal. 2: ra67. doi: 10.1126/scisignal.20 00522

Bernales, S., McDonald, K. L., and Walter, P. (2006). Autophagy counterbalances endoplasmic reticulum expansion during the unfolded protein response. PLoS Biol. 4: e423. doi: 10.1371/journal.pbio.0040423

\section{ACKNOWLEDGMENTS}

We thank Prof. Barbara Zabłocka and Dr. Magdalena Czeredys for critically reading the manuscript.

Berridge, M. J., Lipp, P., and Bootman, M. D. (2000). The versatility and universality of calcium signalling. Nat. Rev. Mol. Cell Biol. 1, 11-21. doi: $10.1038 / 35036035$

Blaustein, M. P., and Golovina, V. A. (2001). Structural complexity and functional diversity of endoplasmic reticulum $\mathrm{Ca}(2+)$ stores. Trends Neurosci. 24, 602-608. doi: 10.1016/S0166-2236(00)01891-9

Blaustein, M. P., Juhaszova, M., Golovina, V. A., Church, P. J., and Stanley, E. F. (2002). Na/Ca exchanger and PMCA localization in neurons and astrocytes: functional implications. Ann. N. Y. Acad. Sci. 976, 356-366. doi: 10.1111/j.1749-6632.2002.tb04762.x

Bojarski, L., Pomorski, P., Szybinska, A., Drab, M., Skibinska-Kijek, A., Gruszczynska-Biegala, J., et al. (2009). Presenilin-dependent expression of STIM proteins and dysregulation of capacitative $\mathrm{Ca} 2+$ entry in familial Alzheimer's disease. Biochim. Biophys. Acta 1793, 1050-1057. doi: 10.1016/j.bbamcr.2008.11.008

Bollimuntha, S., Ebadi, M., and Singh, B. B. (2006). TRPC1 protects human SHSY5Y cells against salsolinol-induced cytotoxicity by inhibiting apoptosis. Brain Res. 1099, 141-149. doi: 10.1016/j.brainres.2006.04.104

Boutillier, S., Lannes, B., Buée, L., Delacourte, A., Rouaux, C., Mohr, M., et al. (2007). Sp3 and sp4 transcription factor levels are increased in brains of patients with Alzheimer's disease. Neurodegener. Dis. 4, 413-423. doi: 10.1159/000107701

Brandman, O., Liou, J., Park, W. S., and Meyer, T. (2007). STIM2 is a feedback regulator that stabilizes basal cytosolic and endoplasmic reticulum $\mathrm{Ca}^{2+}$ levels. Cell 131, 1327-1339. doi: 10.1016/j.cell.2007.11.039

Brini, M., Cal,ì, T., Ottolini, D., and Carafoli, E. (2014). Neuronal calcium signaling: function and dysfunction. Cell. Mol. Life Sci. 71, 2787-2814. doi: 10.1007/s00018-013-1550-7

Brini, M., and Carafoli, E. (2011). The plasma membrane $\mathrm{Ca}^{2}+$ ATPase and the plasma membrane sodium calcium exchanger cooperate in the regulation of cell calcium. Cold. Spring Harb. Perspect. Biol. 3:a004168. doi: $10.1101 /$ cshperspect.a004168

Britzolaki, A., Saurine, J., Klocke, B., and Pitychoutis, P. M. (2020). A role for SERCA pumps in the neurobiology of neuropsychiatric and neurodegenerative disorders. Adv. Exp. Med. Biol. 1131, 131-161. doi: 10.1007/978-3-030-12457-1_6

Brockhaus, J., Schreitmüller, M., Repetto, D., Klatt, O., Reissner, C., Elmslie, K., et al. (2018). $\alpha$-neurexins together with $\alpha 2 \delta$-1 auxiliary subunits regulate $\mathrm{Ca}^{2+}$ influx through Ca v 2.1 channels. J. Neurosci. 38, 8277-8294. doi: 10.1523/JNEUROSCI.0511-18.2018

Campiglio, M., and Flucher, B. E. (2015). The role of auxiliary subunits for the functional diversity of voltage-gated calcium channels. J. Cell. Physiol. 230, 2019-2031. doi: 10.1002/jcp.24998

Cárdenas, C., Miller, R. A., Smith, I., Bui, T., Molgó, J., Müller, M., et al. (2010). Essential regulation of cell bioenergetics by constitutive InsP3 receptor $\mathrm{Ca}^{2+}$ transfer to mitochondria. Cell 142, 270-283. doi: 10.1016/j.cell.2010.06.007

Chakraborty, S., Deb, B. K., Chorna, T., Konieczny, V., Taylor, C. W., and Hasan, G. (2016). Mutant IP3 receptors attenuate store-operated $\mathrm{Ca}^{2+}$ entry by destabilizing STIM-Orai interactions in Drosophila neurons. J. Cell Sci. 129, 3903-3910. doi: 10.1242/jcs. 191585

Chakraborty, S., and Hasan, G. (2017). Spontaneous $\mathrm{Ca}^{2+}$ influx in drosophila pupal neurons is modulated by IP3receptor function and influences maturation of the flight circuit. Front. Mol. Neurosci. 10:111. doi: 10.3389/fnmol.2017. 00111 
Chakroborty, S., and Stutzmann, G. E. (2014). Calcium channelopathies and Alzheimer's disease: insight into therapeutic success and failures. Eur. J. Pharmacol. 739, 83-95. doi: 10.1016/j.ejphar.2013.11.012

Chang, C. L., Chen, Y. J., Quintanilla, C. G., Hsieh, T. S., and Liou, J. (2018). EB1 binding restricts STIM1 translocation to ER-PM junctions and regulates storeoperated Ca entry. J. Cell Biol. 217, 2047-2058. doi: 10.1083/jcb.201711151

Chen, T., Fei, F., Jiang, X. F., Zhang, L., Qu, Y., Huo, K., et al. (2012). Downregulation of Homer $1 \mathrm{~b} / \mathrm{c}$ attenuates glutamate-mediated excitotoxicity through endoplasmic reticulum and mitochondria pathways in rat cortical neurons. Free Radic. Biol. Med. 52, 208-217. doi: 10.1016/j.freeradbiomed.2011.10.451

Coe, H., and Michalak, M. (2010). ERp57, a multifunctional endoplasmic reticulum resident oxidoreductase. Int. J. Biochem. Cell Biol. 42, 796-799. doi: 10.1016/j.biocel.2010.01.009

Cottrell, G. S., Soubrane, C. H., Hounshell, J. A., Lin, H., Owenson, V., Rigby, M., et al. (2018). CACHD1 is an $\alpha 2 \delta$-like protein that modulates Ca V 3 voltage-gated calcium channel activity. J. Neurosci. 38, 9186-9201. doi: 10.1523/JNEUROSCI.3572-15.2018

Crupi, R., Impellizzeri, D., and Cuzzocrea, S. (2019). Role of metabotropic glutamate receptors in neurological disorders. Front. Mol. Neurosci. 12:20. doi: $10.3389 /$ fnmol.2019.00020

Cull-Candy, S. G., and Leszkiewicz, D. N. (2004). Role of distinct NMDA receptor subtypes at central synapses. Sci. STKE 2004: re16. doi: 10.1126/stke.2552004re16

Czeredys, M., Vigont, V. A., Boeva, V. A., Mikoshiba, K., Kaznacheyeva, E. V., and Kuznicki, J. (2018). Huntingtin-associated protein 1A regulates store-operated calcium entry in medium spiny neurons from transgenic YAC128 mice, a model of Huntington's disease. Front. Cell. Neurosci. 12:381. doi: 10.3389/fncel.2018.00381

Dahimene, S., Page, K. M., Kadurin, I., Ferron, L., Ho, D. Y., Powell, G. T., et al. (2018). The $\alpha 2 \delta$-like protein cachd 1 increases $\mathrm{N}$-type calcium currents and cell surface expression and competes with $\alpha 2 \delta$-1. Cell Rep. 25, 1610.e5-1621.e5. doi: 10.1016/j.celrep.2018.10.033

Darbellay, B., Arnaudeau, S., Bader, C. R., Konig, S., and Bernheim, L. (2011). STIM1L is a new actin-binding splice variant involved in fast repetitive $\mathrm{Ca}^{2+}$ release. J. Cell Biol. 194, 335-346. doi: 10.1083/jcb.201012157

Deb, B. K., Chakraborty, P., Gopurappilly, R., and Hasan, G. (2020). SEPT7 regulates $\mathrm{Ca}^{2+}$ entry through Orai channels in human neural progenitor cells and neurons. Cell Calcium 90:102252. doi: 10.1016/j.ceca.2020.102252

Deb, B. K., and Hasan, G. (2016). Regulation of store-operated $\mathrm{Ca}^{2+}$ entry by septins. Front. Cell Dev. Biol. 4:142. doi: 10.3389/fcell.2016.00142

Deb, B. K., and Hasan, G. (2019). SEPT7-mediated regulation of Ca2+ entry through Orai channels requires other septin subunits. Cytoskeleton 76, 104-114. doi: 10.1002/cm.21476

Deb, B. K., Pathak, T., and Hasan, G. (2016). Store-independent modulation of $\mathrm{Ca}(2+)$ entry through Orai by Septin 7. Nat. Commun. 7:11751. doi: $10.1038 /$ ncomms 11751

Deller, T., Korte, M., Chabanis, S., Drakew, A., Schwegler, H., Stefani, G. G., et al. (2003). Synaptopodin-deficient mice lack a spine apparatus and show deficits in synaptic plasticity. Proc. Natl. Acad. Sci. U.S.A. 100, 10494-10499. doi: $10.1073 /$ pnas. 1832384100

Dennis, D. J., Han, S., and Schuurmans, C. (2019). bHLH transcription factors in neural development, disease, and reprogramming. Brain Res. 1705, 48-65. doi: 10.1016/j.brainres.2018.03.013

Derkach, V., Barria, A., and Soderling, T. R. (1999). $\mathrm{Ca}^{2+} /$ calmodulin-kinase II enhances channel conductance of alpha-amino-3-hydroxy-5-methyl-4isoxazolepropionate type glutamate receptors. Proc. Natl. Acad. Sci. U.S.A. 96, 3269-3274. doi: 10.1073/pnas.96.6.3269

Desai, P. N., Zhang, X., Wu, S., Janoshazi, A., Bolimuntha, S., Putney, J. W., et al. (2015). Multiple types of calcium channels arising from alternative translation initiation of the Orail message. Sci. Signal. 8: ra74. doi: 10.1126/scisignal.aaa8323

Di Buduo, C. A., Abbonante, V., Marty, C., Moccia, F., Rumi, E., Pietra, D., et al. (2020). Defective interaction of mutant calreticulin and SOCE in megakaryocytes from patients with myeloproliferative neoplasms. Blood 135, 133-144. doi: 10.1182/blood.2019001103

Dick, O., and Bading, H. (2010). Synaptic activity and nuclear calcium signaling protect hippocampal neurons from death signal-associated nuclear translocation of FoxO3a induced by extrasynaptic N-methyl-D-aspartate receptors. J. Biol. Chem. 285, 19354-19361. doi: 10.1074/jbc.M110.127654

Ding, W. X., Ni, H. M., Gao, W., Hou, Y. F., Melan, M. A., Chen, X., et al. (2007). Differential effects of endoplasmic reticulum stress-induced autophagy on cell survival. J. Biol. Chem. 282, 4702-4710. doi: 10.1074/jbc.M609267200

Dionisio, N., Smani, T., Woodard, G. E., Castellano, A., Salido, G. M., and Rosado, J. A. (2015). Homer proteins mediate the interaction between STIM1 and Cav1.2 channels. Biochim. Biophys. Acta 1853, 1145-1153. doi: 10.1016/j.bbamcr.2015.02.014

Dittmer, P. J., Wild, A. R., Dell'Acqua, M. L., and Sather, W. A. (2017). STIM1 $\mathrm{Ca}^{2+}$ sensor control of L-type $\mathrm{Ca}^{2+}$-channel-dependent dendritic spine structural plasticity and nuclear signaling. Cell Rep. 19, 321-334. doi: 10.1016/j.celrep.2017.03.056

Domenichini, F., Terrié, E., Arnault, P., Harnois, T., Magaud, C., Bois, P., et al. (2018). Store-operated calcium entries control neural stem cell selfrenewal in the adult brain subventricular zone. Stem Cells 36, 761-774. doi: $10.1002 /$ stem.2786

Duszyński, J., Kozie,ł, R., Brutkowski, W., Szczepanowska, J., and Zabłocki, K. (2006). The regulatory role of mitochondria in capacitative calcium entry. Biochim. Biophys. Acta 1757, 380-387. doi: 10.1016/j.bbabio.2006.04.017

Emptage, N., Bliss, T. V., and Fine, A. (1999). Single synaptic events evoke NMDA receptor-mediated release of calcium from internal stores in hippocampal dendritic spines. Neuron 22, 115-124. doi: 10.1016/S0896-6273(00)80683-2

Emptage, N. J., Reid, C. A., and Fine, A. (2001). Calcium stores in hippocampal synaptic boutons mediate short-term plasticity, store-operated $\mathrm{Ca}^{2+}$ entry, and spontaneous transmitter release. Neuron 29, 197-208. doi: 10.1016/S0896-6273(01)00190-8

Esteban, J. A., Shi, S. H., Wilson, C., Nuriya, M., Huganir, R. L., and Malinow, R. (2003). PKA phosphorylation of AMPA receptor subunits controls synaptic trafficking underlying plasticity. Nat. Neurosci. 6, 136-143. doi: 10.1038/nn997

Fierro, L., DiPolo, R., and Llano, I. (1998). Intracellular calcium clearance in Purkinje cell somata from rat cerebellar slices. J. Physiol. 510 (Pt 2), 499-512. doi: 10.1111/j.1469-7793.1998.499bk.x

Filipovic, R., Rakic, S., and Zecevic, N. (2002). Expression of Golli proteins in adult human brain and multiple sclerosis lesions. J. Neuroimmunol. 127, 1-12. doi: 10.1016/S0165-5728(02)00070-X

Fong, A. P., Yao, Z., Zhong, J. W., Cao, Y., Ruzzo, W. L., Gentleman, R. C., et al. (2012). Genetic and epigenetic determinants of neurogenesis and myogenesis. Dev. Cell 22, 721-735. doi: 10.1016/j.devcel.2012.01.015

Fonseca, A. C., Moreira, P. I., Oliveira, C. R., Cardoso, S. M., Pinton, P., and Pereira, C. F. (2015). Amyloid-beta disrupts calcium and redox homeostasis in brain endothelial cells. Mol. Neurobiol. 51, 610-622. doi: $10.1007 / \mathrm{s} 12035-014-8740-7$

Gao, X., Xia, J., Munoz, F. M., Manners, M. T., Pan, R., Meucci, O., et al. (2016). STIMs and Orail regulate cytokine production in spinal astrocytes. J. Neuroinflammation 13:126. doi: 10.1186/s12974-016-0594-7

Garcia-Alvarez, G., Lu, B., Yap, K. A., Wong, L. C., Thevathasan, J. V., Lim, L., et al. (2015). STIM2 regulates PKA-dependent phosphorylation and trafficking of AMPARs. Mol. Biol. Cell 26, 1141-1159. doi: 10.1091/mbc.E14-07-1222

Gemes, G., Bangaru, M. L., Wu, H. E., Tang, Q., Weihrauch, D., Koopmeiners, A. S., et al. (2011). Store-operated $\mathrm{Ca}^{2+}$ entry in sensory neurons: functional role and the effect of painful nerve injury. J. Neurosci. 31, 3536-3549. doi: 10.1523/JNEUROSCI.5053-10.2011

Ghavami, S., Shojaei, S., Yeganeh, B., Ande, S. R., Jangamreddy, J. R., Mehrpour, M., et al. (2014). Autophagy and apoptosis dysfunction in neurodegenerative disorders. Prog. Neurobiol. 112, 24-49. doi: 10.1016/j.pneurobio.2013.10.004

Golovina, V. A. (2005). Visualization of localized store-operated calcium entry in mouse astrocytes. Close proximity to the endoplasmic reticulum. J. Physiol. 564, 737-749. doi: 10.1113/jphysiol.2005.085035

Gopurappilly, R., Deb, B. K., Chakraborty, P., and Hasan, G. (2018). Stable STIM1 knockdown in self-renewing human neural precursors promotes premature neural differentiation. Front. Mol. Neurosci. 11:178. doi: 10.3389/fnmol.2018.00178

Greotti, E., Capitanio, P., Wong, A., Pozzan, T., Pizzo, P., and Pendin, D. (2019). Familial Alzheimer's disease-linked presenilin mutants and intracellular $\mathrm{Ca}^{2+}$ handling: a single-organelle, FRET-based analysis. Cell Calcium 79, 44-56. doi: 10.1016/j.ceca.2019.02.005 
Grigoriev, I., Gouveia, S. M., van der Vaart, B., Demmers, J., Smyth, J. T., Honnappa, S., et al. (2008). STIM1 is a MT-plus-end-tracking protein involved in remodeling of the ER. Curr. Biol. 18, 177-182. doi: 10.1016/j.cub.2007.12.050

Gruszczynska-Biegala, J., and Kuznicki, J. (2013). Native STIM2 and ORAI1 proteins form a calcium-sensitive and thapsigargin-insensitive complex in cortical neurons. J. Neurochem. 126, 727-738. doi: 10.1111/jnc.12320

Gruszczynska-Biegala, J., Pomorski, P., Wisniewska, M. B., and Kuznicki, J. (2011). Differential roles for STIM1 and STIM2 in store-operated calcium entry in rat neurons. PLoS ONE 6: e19285. doi: 10.1371/journal.pone.0019285

Gruszczynska-Biegala, J., Sladowska, M., and Kuznicki, J. (2016). AMPA receptors are involved in store-operated calcium entry and interact with STIM proteins in rat primary cortical neurons. Front. Cell. Neurosci. 10:251. doi: 10.3389/fncel.2016.00251

Gruszczynska-Biegala, J., Strucinska, K., Maciag, F., Majewski, L., Sladowska, M., and Kuznicki, J. (2020). STIM Protein-NMDA2 receptor interaction decreases NMDA-dependent calcium levels in cortical neurons. Cells 9:160. doi: $10.3390 /$ cells 9010160

Gu, J., Firestein, B. L., and Zheng, J. Q. (2008). Microtubules in dendritic spine development. J. Neurosci. 28, 12120-12124. doi: 10.1523/JNEUROSCI.2509-08.2008

Guner, G., Guzelsoy, G., Isleyen, F. S., Sahin, G. S., Akkaya, C., Bayam, E., et al. (2017). NEUROD2 regulates stim1 expression and store-operated calcium entry in cortical neurons. eNeuro 4, 1-17. doi: 10.1523/ENEURO.0255-16.2017

Harraz, O. F., and Altier, C. (2014). STIM1-mediated bidirectional regulation of $\mathrm{Ca}(2+)$ entry through voltage-gated calcium channels (VGCC) and calcium-release activated channels (CRAC). Front. Cell Neurosci. 8:43. doi: $10.3389 /$ fncel.2014.00043

Hartmann, J., Dragicevic, E., Adelsberger, H., Henning, H. A., Sumser, M., Abramowitz, J., et al. (2008). TRPC3 channels are required for synaptic transmission and motor coordination. Neuron 59, 392-398. doi: 10.1016/j.neuron.2008.06.009

Hartmann, J., Karl, R. M., Alexander, R. P., Adelsberger, H., Brill, M. S., Rühlmann, C., et al. (2014). STIM1 controls neuronal $\mathrm{Ca}^{2}+$ signaling, mGluR1-dependent synaptic transmission, and cerebellar motor behavior. Neuron 82, 635-644. doi: 10.1016/j.neuron.2014.03.027

Hawkins, B. J., Irrinki, K. M., Mallilankaraman, K., Lien, Y. C., Wang, Y., Bhanumathy, C. D., et al. (2010). S-glutathionylation activates STIM1 and alters mitochondrial homeostasis. J. Cell Biol. 190, 391-405. doi: $10.1083 /$ jcb. 201004152

Heine, M., Heck, J., Ciuraszkiewicz, A., and Bikbaev, A. (2020). Dynamic compartmentalization of calcium channel signalling in neurons. Neuropharmacology 169:107556. doi: 10.1016/j.neuropharm.2019.02.038

Henke, N., Albrecht, P., Pfeiffer, A., Toutzaris, D., Zanger, K., and Methner, A. (2012). Stromal interaction molecule 1 (STIM1) is involved in the regulation of mitochondrial shape and bioenergetics and plays a role in oxidative stress. $J$. Biol. Chem. 287, 42042-42052. doi: 10.1074/jbc.M112.417212

Heo, D. K., Lim, H. M., Nam, J. H., Lee, M. G., and Kim, J. Y. (2015). Regulation of phagocytosis and cytokine secretion by store-operated calcium entry in primary isolated murine microglia. Cell. Signal. 27, 177-186. doi: $10.1016 /$ j.cellsig.2014.11.003

Herms, J., Schneider, I., Dewachter, I., Caluwaerts, N., Kretzschmar, H., and Van Leuven, F. (2003). Capacitive calcium entry is directly attenuated by mutant presenilin-1, independent of the expression of the amyloid precursor protein. J. Biol. Chem. 278, 2484-2489. doi: 10.1074/jbc.M206769200

Hong, J. H., Li, Q., Kim, M. S., Shin, D. M., Feske, S., Birnbaumer, L., et al. (2011). Polarized but differential localization and recruitment of STIM1, Orail and TRPC channels in secretory cells. Traffic 12, 232-245. doi: 10.1111/j.1600-0854.2010.01138.x

Honnappa, S., Gouveia, S. M., Weisbrich, A., Damberger, F. F., Bhavesh, N. S., Jawhari, H., et al. (2009). An EB1-binding motif acts as a microtubule tip localization signal. Cell 138, 366-376. doi: 10.1016/j.cell.2009.04.065

Hoth, M., and Niemeyer, B. A. (2013). The neglected CRAC proteins: Orai2, Orai3, and STIM2. Curr. Top. Membr. 71, 237-271. doi: 10.1016/B978-0-12-407870-3.00010-X

Høyer-Hansen, M., Bastholm, L., Szyniarowski, P., Campanella, M., Szabadkai, G., Farkas, T., et al. (2007). Control of macroautophagy by calcium, calmodulin-dependent kinase kinase-beta, and Bcl-2. Mol. Cell 25, 193-205. doi: 10.1016/j.molcel.2006.12.009
Høyer-Hansen, M., and Jäättelä, M. (2007). Connecting endoplasmic reticulum stress to autophagy by unfolded protein response and calcium. Cell Death Differ. 14, 1576-1582. doi: 10.1038/sj.cdd.4402200

Hu, X., Viesselmann, C., Nam, S., Merriam, E., and Dent, E. W. (2008). Activitydependent dynamic microtubule invasion of dendritic spines. J. Neurosci. 28, 13094-13105. doi: 10.1523/JNEUROSCI.3074-08.2008

Hu, Y. D., Tang, C. L., Jiang, J. Z., Lv, H. Y., Wu, Y. B., Qin, X. D., et al. (2020). Neuroprotective effects of dexmedetomidine preconditioning on oxygen-glucose deprivation-reoxygenation injury in $\mathrm{PC} 12$ cells via regulation of $\mathrm{Ca}^{2+}$-STIM1/Orail signaling. Curr. Med. Sci. 40, 699-707. doi: 10.1007/s11596-020-2201-5

Ivanova, H., Vervliet, T., Missiaen, L., Parys, J. B., De Smedt, H., and Bultynck, G. (2014). Inositol 1,4,5-trisphosphate receptor-isoform diversity in cell death and survival. Biochim. Biophys. Acta 1843, 2164-2183. doi: 10.1016/j.bbamcr.2014.03.007

Jardin, I., Albarrán, L., Bermejo, N., Salido, G. M., and Rosado, J. A. (2012). Homers regulate calcium entry and aggregation in human platelets: a role for Homers in the association between STIM1 and Orail. Biochem. J. 445, 29-38. doi: 10.1042/BJ20120471

Jha, A., Ahuja, M., Maléth, J., Moreno, C. M., Yuan, J. P., Kim, M. S., et al. (2013). The STIM1 CTID domain determines access of SARAF to SOAR to regulate Orail channel function. J. Cell Biol. 202, 71-79. doi: 10.1083/jcb.201301148

Katz, Z. B., Zhang, C., Quintana, A., Lillemeier, B. F., and Hogan, P. G. (2019). Septins organize endoplasmic reticulum-plasma membrane junctions for STIM1-ORAI1 calcium signalling. Sci. Rep. 9:10839. doi: 10.1038/s41598-019-46862-w

Keil, J. M., Shen, Z., Briggs, S. P., and Patrick, G. N. (2010). Regulation of STIM1 and SOCE by the ubiquitin-proteasome system (UPS). PLOS ONE 5:e13465. doi: 10.1371/journal.pone.0013465

Kerchner, G. A., and Nicoll, R. A. (2008). Silent synapses and the emergence of a postsynaptic mechanism for LTP. Nat. Rev. Neurosci. 9, 813-825. doi: $10.1038 / \mathrm{nrn} 2501$

Kettenmann, H. R., and Bruce, R. (2013). Neuroglia. Oxford: Oxford University Press. doi: 10.1093/med/9780199794591.001.0001

Klejman, M. E., Gruszczynska-Biegala, J., Skibinska-Kijek, A., Wisniewska, M. B., Misztal, K., Blazejczyk, M., et al. (2009). Expression of STIM1 in brain and puncta-like co-localization of STIM1 and ORAI1 upon depletion of $\mathrm{Ca}(2+)$ store in neurons. Neurochem. Int. 54, 49-55. doi: 10.1016/j.neuint.2008.10.005

Kondratskyi, A., Yassine, M., Slomianny, C., Kondratska, K., Gordienko, D., Dewailly, E., et al. (2014). Identification of ML-9 as a lysosomotropic agent targeting autophagy and cell death. Cell Death Dis. 5:e1193. doi: $10.1038 /$ cddis.2014.156

Korkotian, E., Frotscher, M., and Segal, M. (2014). Synaptopodin regulates spine plasticity: mediation by calcium stores. J. Neurosci. 34, 11641-11651. doi: 10.1523/JNEUROSCI.0381-14.2014

Korkotian, E., Oni-Biton, E., and Segal, M. (2017). The role of the store-operated calcium entry channel Orail in cultured rat hippocampal synapse formation and plasticity. J. Physiol. 595, 125-140. doi: 10.1113/JP272645

Kraft, R. (2015). STIM and ORAI proteins in the nervous system. Channels 9, 245-252. doi: 10.1080/19336950.2015.1071747

Krapivinsky, G., Krapivinsky, L., Stotz, S. C., Manasian, Y., and Clapham, D. E. (2011). POST, partner of stromal interaction molecule 1 (STIM1), targets STIM1 to multiple transporters. Proc. Natl. Acad. Sci. U.S.A. 108, 19234-19239. doi: 10.1073/pnas. 1117231108

Kroemer, G., Mariño, G., and Levine, B. (2010). Autophagy and the integrated stress response. Mol. Cell 40, 280-293. doi: 10.1016/j.molcel.2010. 09.023

Kuang, X. L., Liu, Y., Chang, Y., Zhou, J., Zhang, H., Li, Y., et al. (2016). Inhibition of store-operated calcium entry by sub-lethal levels of proteasome inhibition is associated with STIM1/STIM2 degradation. Cell Calcium 59, 172-180. doi: 10.1016/j.ceca.2016.01.007

Kumar, U., Dunlop, D. M., and Richardson, J. S. (1994). Mitochondria from Alzheimer's fibroblasts show decreased uptake of calcium and increased sensitivity to free radicals. Life Sci. 54, 1855-1860. doi: 10.1016/0024-3205(94)90142-2

Kwon, J., An, H., Sa, M., Won, J., Shin, J. I., and Lee, C. J. (2017). Orail and Orai3 in combination with stim 1 mediate the majority of store-operated calcium entry in astrocytes. Exp. Neurobiol. 26, 42-54. doi: 10.5607/en.2017.26.1.42 
La Russa, D., Frisina, M., Secondo, A., Bagetta, G., and Amantea, D. (2020). Modulation of cerebral store-operated calcium entryregulatory factor (SARAF) and peripheral Orail following focal cerebral ischemia and preconditioning in mice. Neuroscience 441, 8-21. doi: 10.1016/j.neuroscience.2020.06.014

Lalonde, J., Saia, G., and Gill, G. (2014). Store-operated calcium entry promotes the degradation of the transcription factor Sp4 in resting neurons. Sci. Signal. 7: ra51. doi: 10.1126/scisignal.2005242

Law, B. Y., Wang, M., Ma, D. L., Al-Mousa, F., Michelangeli, F., Cheng, S. H., et al. (2010). Alisol, B., a novel inhibitor of the sarcoplasmic/endoplasmic reticulum $\mathrm{Ca}(2+)$ ATPase pump, induces autophagy, endoplasmic reticulum stress, and apoptosis. Mol. Cancer Ther. 9, 718-730. doi: 10.1158/1535-7163.MCT-09-0700

Lee, K. P., Yuan, J. P., Zeng, W., So, I., Worley, P. F., and Muallem, S. (2009). Molecular determinants of fast $\mathrm{Ca}^{2+}$-dependent inactivation and gating of the Orai channels. Proc. Natl. Acad. Sci. U.S.A. 106, 14687-14692. doi: 10.1073/pnas.0904664106

LeMaistre, J. L., Sanders, S. A., Stobart, M. J., Lu, L., Knox, J. D., Anderson, H. D., et al. (2012). Coactivation of NMDA receptors by glutamate and D-serine induces dilation of isolated middle cerebral arteries. J. Cereb. Blood Flow Metab. 32, 537-547. doi: 10.1038/jcbfm.2011.161

Li, X., Chen, W., Zhang, L., Liu, W. B., and Fei, Z. (2013). Inhibition of store-operated calcium entry attenuates $\mathrm{MPP}(+)$-induced oxidative stress via preservation of mitochondrial function in PC12 cells: involvement of Homerla. PLoS ONE 8: e83638. doi: 10.1371/journal.pone.0083638

Li, Y., and Camacho, P. (2004). $\mathrm{Ca}^{2+}$-dependent redox modulation of SERCA $2 \mathrm{~b}$ by ERp57. J. Cell Biol. 164, 35-46. doi: 10.1083/jcb.200307010

Liao, Y., Erxleben, C., Abramowitz, J., Flockerzi, V., Zhu, M. X., Armstrong, D. L., et al. (2008). Functional interactions among Orai1, TRPCs, and STIM1 suggest a STIM-regulated heteromeric Orai/TRPC model for SOCE/Icrac channels. Proc. Natl. Acad. Sci. U.S.A. 105, 2895-2900. doi: 10.1073/pnas.0712288105

Lim, D., Mapelli, L., Canonico, P. L., Moccia, F., and Genazzani, A. A. (2018). Neuronal activity-dependent activation of astroglial calcineurin in mouse primary hippocampal cultures. Int. J. Mol. Sci. 19:2997. doi: $10.3390 /$ ijms 19102997

Liou, J., Kim, M. L., Heo, W. D., Jones, J. T., Myers, J. W., Ferrell, J. E., et al. (2005). STIM is a $\mathrm{Ca}^{2+}$ sensor essential for $\mathrm{Ca}^{2+}$-store-depletion-triggered $\mathrm{Ca}^{2+}$ influx. Curr. Biol. 15, 1235-1241. doi: 10.1016/j.cub.2005.05.055

López, J. J., Salido, G. M., Pariente, J. A., and Rosado, J. A. (2006). Interaction of STIM1 with endogenously expressed human canonical TRP1 upon depletion of intracellular $\mathrm{Ca}^{2+}$ stores. J. Biol. Chem. 281, 28254-28264. doi: 10.1074/jbc.M604272200

Luo, P., Li, X., Fei, Z., and Poon, W. (2012). Scaffold protein Homer 1: implications for neurological diseases. Neurochem. Int. 61, 731-738. doi: 10.1016/j.neuint.2012.06.014

Lur, G., Sherwood, M. W., Ebisui, E., Haynes, L., Feske, S., Sutton, R., et al. (2011). InsP3receptors and Orai channels in pancreatic acinar cells: co-localization and its consequences. Biochem. J. 436, 231-239. doi: 10.1042/BJ20110083

Majewski, L., and Kuznicki, J. (2015). SOCE in neurons: Signaling or just refilling? Biochim. Biophys. Acta 1853, 1940-1952. doi: 10.1016/j.bbamcr.2015.01.019

Majewski, Ł., Maciag, F., Boguszewski, P. M., Wasilewska, I., Wiera, G., Wójtowicz, T., et al. (2017). Overexpression of STIM1 in neurons in mouse brain improves contextual learning and impairs long-term depression. Biochim. Biophys. Acta Mol. Cell Res. 1864, 1071-1087. doi: 10.1016/j.bbamcr.2016.11.025

Makino, H., and Malinow, R. (2011). Compartmentalized versus global synaptic plasticity on dendrites controlled by experience. Neuron 72, 1001-1011. doi: 10.1016/j.neuron.2011.09.036

Malarkey, E. B., Ni, Y., and Parpura, V. (2008). $\mathrm{Ca}^{2+}$ entry through TRPC1 channels contributes to intracellular $\mathrm{Ca}^{2+}$ dynamics and consequent glutamate release from rat astrocytes. Glia 56, 821-835. doi: 10.1002/glia.20656

Malenka, R. C., and Bear, M. F. (2004). LTP and LTD: an embarrassment of riches. Neuron 44, 5-21. doi: 10.1016/j.neuron.2004.09.012

Marambaud, P., Dreses-Werringloer, U., and Vingtdeux, V. (2009). Calcium signaling in neurodegeneration. Mol. Neurodegener. 4:20. doi: 10.1186/1750-1326-4-20

Marttinen, M., Kurkinen, K. M., Soininen, H., Haapasalo, A., and Hiltunen, M. (2015). Synaptic dysfunction and septin protein family members in neurodegenerative diseases. Mol. Neurodegener. 10:16. doi: $10.1186 / \mathrm{s} 13024-015-0013-\mathrm{z}$
Mavrakis, M., Azou-Gros, Y., Tsai, F. C., Alvarado, J., Bertin, A., Iv, F., et al. (2014). Septins promote F-actin ring formation by crosslinking actin filaments into curved bundles. Nat. Cell Biol. 16, 322-334. doi: 10.1038/ncb2921

Mercer, J. C., Dehaven, W. I., Smyth, J. T., Wedel, B., Boyles, R. R., Bird, G. S., et al. (2006). Large store-operated calcium selective currents due to co-expression of Orai1 or Orai2 with the intracellular calcium sensor, Stim1. J. Biol. Chem. 281, 24979-24990. doi: 10.1074/jbc.M604589200

Michaelis, M., Nieswandt, B., Stegner, D., Eilers, J., and Kraft, R. (2015). STIM1, STIM2, and Orail regulate store-operated calcium entry and purinergic activation of microglia. Glia 63, 652-663. doi: 10.1002/glia.22775

Miyano, K., Morioka, N., Sugimoto, T., Shiraishi, S., Uezono, Y., and Nakata, Y. (2010). Activation of the neurokinin-1 receptor in rat spinal astrocytes induces $\mathrm{Ca}^{2+}$ release from IP3-sensitive $\mathrm{Ca}^{2+}$ stores and extracellular $\mathrm{Ca}^{2+}$ influx through TRPC3. Neurochem. Int. 57, 923-934. doi: 10.1016/j.neuint.2010.09.012

Moccia, F., Zuccolo, E., Soda, T., Tanzi, F., Guerra, G., Mapelli, L., et al. (2015). Stim and Orai proteins in neuronal $\mathrm{Ca}(2+)$ signaling and excitability. Front. Cell Neurosci. 9:153. doi: 10.3389/fncel.2015.00153

Molnár, T., Yarishkin, O., Iuso, A., Barabas, P., Jones, B., Marc, R. E., et al. (2016). Store-operated calcium entry in Müller Glia is controlled by synergistic activation of TRPC and orai channels. J. Neurosci. 36, 3184-3198. doi: 10.1523/JNEUROSCI.4069-15.2016

Moreno, C., Sampieri, A., Vivas, O., Peña-Segura, C., and Vaca, L. (2012). STIM1 and Orail mediate thrombin-induced $\mathrm{Ca}(2+)$ influx in rat cortical astrocytes. Cell Calcium 52, 457-467. doi: 10.1016/j.ceca.2012.08.004

Moscarello, M. A., Mak, B., Nguyen, T. A., Wood, D. D., Mastronardi, F., and Ludwin, S. K. (2002). Paclitaxel (Taxol) attenuates clinical disease in a spontaneously demyelinating transgenic mouse and induces remyelination. Mult. Scler 8, 130-138. doi: 10.1191/1352458502ms776oa

Mostowy, S., and Cossart, P. (2012). Septins: the fourth component of the cytoskeleton. Nat. Rev. Mol. Cell Biol. 13, 183-194. doi: 10.1038/nrm3284

Motiani, R. K., Hyzinski-García, M. C., Zhang, X., Henkel, M. M., Abdullaev, I. F., Kuo, Y. H., et al. (2013). STIM1 and Orail mediate CRAC channel activity and are essential for human glioblastoma invasion. Pflugers Arch. 465, 1249-1260. doi: 10.1007/s00424-013-1254-8

Muik, M., Fahrner, M., Derler, I., Schindl, R., Bergsmann, J., Frischauf, I., et al. (2009). A cytosolic homomerization and a modulatory domain within STIM1 C terminus determine coupling to ORAI1 channels. J. Biol. Chem. 284, 8421-8426. doi: 10.1074/jbc.C800229200

Nakazawa, K., McHugh, T. J., Wilson, M. A., and Tonegawa, S. (2004). NMDA receptors, place cells and hippocampal spatial memory. Nat. Rev. Neurosci. 5, 361-372. doi: 10.1038/nrn1385

Negri, S., Faris, P., Pellavio, G., Botta, L., Orgiu, M., Forcaia, G., et al. (2020). Group 1 metabotropic glutamate receptors trigger glutamate-induced intracellular $\mathrm{Ca}^{2+}$ signals and nitric oxide release in human brain microvascular endothelial cells. Cell. Mol. Life Sci. 77, 2235-2253. doi: 10.1007/s00018-019-03284-1

Ng, A. N., Krogh, M., and Toresson, H. (2011). Dendritic EGFP-STIM1 activation after type I metabotropic glutamate and muscarinic acetylcholine receptor stimulation in hippocampal neuron. J. Neurosci. Res. 89, 1235-1244. doi: $10.1002 / j n r .22648$

Ohana, L., Newell, E. W., Stanley, E. F., and Schlichter, L. C. (2009). The $\mathrm{Ca}^{2+}$ release-activated $\mathrm{Ca}^{2+}$ current (I(CRAC)) mediates store-operated $\mathrm{Ca}^{2+}$ entry in rat microglia. Channels 3, 129-139. doi: 10.4161/chan.3. 2.8609

Paez, P. M., Fulton, D., Colwell, C. S., and Campagnoni, A. T. (2009b). Voltageoperated $\mathrm{Ca}(2+)$ and $\mathrm{Na}(+)$ channels in the oligodendrocyte lineage. $J$. Neurosci. Res. 87, 3259-3266. doi: 10.1002/jnr.21938

Paez, P. M., Fulton, D., Spreuer, V., Handley, V., and Campagnoni, A. T. (2011). Modulation of canonical transient receptor potential channel 1 in the proliferation of oligodendrocyte precursor cells by the golli products of the myelin basic protein gene. J. Neurosci. 31, 3625-3637. doi: 10.1523/JNEUROSCI.4424-10.2011

Paez, P. M., Fulton, D. J., Spreuer, V., Handley, V., Campagnoni, C. W., and Campagnoni, A. T. (2009a). Regulation of store-operated and voltage-operated $\mathrm{Ca}^{2+}$ channels in the proliferation and death of oligodendrocyte precursor cells by golli proteins. ASN Neuro 1.e00003. doi: 10.1042/AN20090003

Paez, P. M., Spreuer, V., Handley, V., Feng, J. M., Campagnoni, C., and Campagnoni, A. T. (2007). Increased expression of golli myelin basic 
proteins enhances calcium influx into oligodendroglial cells. J. Neurosci. 27, 12690-12699. doi: 10.1523/JNEUROSCI.2381-07.2007

Palty, R., Raveh, A., Kaminsky, I., Meller, R., and Reuveny, E. (2012). SARAF inactivates the store operated calcium entry machinery to prevent excess calcium refilling. Cell 149, 425-438. doi: 10.1016/j.cell.2012.01.055

Papanikolaou, M., Lewis, A., and Butt, A. M. (2017). Store-operated calcium entry is essential for glial calcium signalling in CNS white matter. Brain Struct. Funct. 222, 2993-3005. doi: 10.1007/s00429-017-1380-8

Park, C. Y., Hoover, P. J., Mullins, F. M., Bachhawat, P., Covington, E. D., Raunser, S., et al. (2009). STIM1 clusters and activates CRAC channels via direct binding of a cytosolic domain to Orail. Cell 136, 876-890. doi: 10.1016/j.cell.2009.02.014

Park, C. Y., Shcheglovitov, A., and Dolmetsch, R. (2010). The CRAC channel activator STIM1 binds and inhibits L-type voltage-gated calcium channels. Science 330, 101-105. doi: 10.1126/science.1191027

Parys, J. B., Decuypere, J. P., and Bultynck, G. (2012). Role of the inositol 1,4,5trisphosphate receptor/ $\mathrm{Ca}^{2+}$-release channel in autophagy. Cell Commun. Signal. 10:17. doi: 10.1186/1478-811X-10-17

Pascual-Caro, C., Berrocal, M., Lopez-Guerrero, A. M., Alvarez-Barrientos, A., Pozo-Guisado, E., Gutierrez-Merino, C., et al. (2018). STIM1 deficiency is linked to Alzheimer's disease and triggers cell death in SH-SY5Y cells by upregulation of L-type voltage-operated Ca. J. Mol. Med. 96, 1061-1079. doi: 10.1007/s00109-018-1677-y

Pascual-Caro, C., Orantos-Aguilera, Y., Sanchez-Lopez, I., de Juan-Sanz, J., Parys, J. B., Area-Gomez, E., et al. (2020). STIM1 deficiency leads to specific down-regulation of ITPR3 in SH-SY5Y cells. Int. J. Mol. Sci. 21:6598. doi: $10.3390 /$ ijms 21186598

Pavez, M., Thompson, A. C., Arnott, H. J., Mitchell, C. B., D'Atri, I., Don, E. K., et al. (2019). STIM1 is required for remodeling of the endoplasmic reticulum and microtubule cytoskeleton in steering growth cones. J. Neurosci. 39, 5095-5114. doi: 10.1523/JNEUROSCI.2496-18.2019

Pchitskaya, E., Kraskovskaya, N., Chernyuk, D., Popugaeva, E., Zhang, H., Vlasova, O., et al. (2017). Stim2-Eb3 association and morphology of dendritic spines in hippocampal neurons. Sci. Rep. 7:17625. doi: 10.1038/s41598-017-17762-8

Pchitskaya, E., Popugaeva, E., and Bezprozvanny, I. (2018). Calcium signaling and molecular mechanisms underlying neurodegenerative diseases. Cell Calcium 70, 87-94. doi: 10.1016/j.ceca.2017.06.008

Pelucchi, S., Stringhi, R., and Marcello, E. (2020). Dendritic spines in alzheimer's disease: how the actin cytoskeleton contributes to synaptic failure. Int. J. Mol. Sci. 21:908. doi: 10.3390/ijms21030908

Pinacho, R., Villalmanzo, N., Lalonde, J., Haro, J. M., Meana, J. J., Gill, G., et al. (2011). The transcription factor SP4 is reduced in postmortem cerebellum of bipolar disorder subjects: control by depolarization and lithium. Bipolar Disord. 13, 474-485. doi: 10.1111/j.1399-5618.2011.00941.x

Popugaeva, E., Chernyuk, D., and Bezprozvanny, I. (2020). Reversal of calcium dysregulation as potential approach for treating Alzheimer's disease. Curr. Alzheimer Res. 17, 344-354. doi: 10.2174/15672050176662005281 62046

Popugaeva, E., Pchitskaya, E., Speshilova, A., Alexandrov, S., Zhang, H., Vlasova, O., et al. (2015). STIM2 protects hippocampal mushroom spines from amyloid synaptotoxicity. Mol. Neurodegener. 10:37. doi: 10.1186/s13024-015-0034-7

Prakriya, M., Feske, S., Gwack, Y., Srikanth, S., Rao, A., and Hogan, P. G. (2006). Orail is an essential pore subunit of the CRAC channel. Nature 443, 230-233. doi: $10.1038 /$ nature 05122

Prins, D., Groenendyk, J., Touret, N., and Michalak, M. (2011). Modulation of STIM1 and capacitative $\mathrm{Ca}^{2+}$ entry by the endoplasmic reticulum luminal oxidoreductase ERp57. EMBO Rep. 12, 1182-1188. doi: $10.1038 /$ embor.2011.173

Putney, J. W. (2003). Capacitative calcium entry in the nervous system. Cell Calcium 34, 339-344. doi: 10.1016/S0143-4160(03)00143-X

Rae, M. G., Martin, D. J., Collingridge, G. L., and Irving, A. J. (2000). Role of $\mathrm{Ca}^{2+}$ stores in metabotropic L-glutamate receptor-mediated supralinear $\mathrm{Ca}^{2+}$ signaling in rat hippocampal neurons. J. Neurosci. 20, 8628-8636. doi: 10.1523/JNEUROSCI.20-23-08628.2000

Rao, W., Peng, C., Zhang, L., Su, N., Wang, K., Hui, H., et al. (2016). Homerla attenuates glutamate-induced oxidative injury in HT-22 cells through regulation of store-operated calcium entry. Sci. Rep. 6:33975. doi: $10.1038 /$ srep 33975
Rao, W., Zhang, L., Peng, C., Hui, H., Wang, K., Su, N., et al. (2015). Downregulation of STIM2 improves neuronal survival after traumatic brain injury by alleviating calcium overload and mitochondrial dysfunction. Biochim. Biophys. Acta 1852, 2402-2413. doi: 10.1016/j.bbadis.2015.08.014

Rao, W., Zhang, L., Su, N., Wang, K., Hui, H., Wang, L., et al. (2013). Blockade of SOCE protects HT22 cells from hydrogen peroxide-induced apoptosis. Biochem. Biophys. Res. Commun. 441, 351-356. doi: 10.1016/j.bbrc.2013.10.054

Remondelli, P., and Renna, M. (2017). The endoplasmic reticulum unfolded protein response in neurodegenerative disorders and its potential therapeutic significance. Front. Mol. Neurosci. 10:187. doi: 10.3389/fnmol.2017.00187

Ritchie, M. F., Samakai, E., and Soboloff, J. (2012). STIM1 is required for attenuation of PMCA-mediated $\mathrm{Ca}^{2+}$ clearance during T-cell activation. EMBO J. 31, 1123-1133. doi: 10.1038/emboj.2011.495

Rogawski, M. A. (2011). Revisiting AMPA receptors as an antiepileptic drug target. Epilepsy Curr. 11, 56-63. doi: 10.5698/1535-7511-11.2.56

Ronco, V., Grolla, A. A., Glasnov, T. N., Canonico, P. L., Verkhratsky, A., Genazzani, A. A., et al. (2014). Differential deregulation of astrocytic calcium signalling by amyloid- $\beta$, TNF $\alpha$, IL-1 $\beta$ and LPS. Cell Calcium 55, 219-229. doi: $10.1016 /$ j.ceca.2014.02.016

Roos, J., DiGregorio, P. J., Yeromin, A. V., Ohlsen, K., Lioudyno, M., Zhang, S., et al. (2005). STIM1, an essential and conserved component of store-operated $\mathrm{Ca}^{2+}$ channel function. J. Cell Biol. 169, 435-445. doi: 10.1083/jcb.200502019

Ryazantseva, M., Goncharova, A., Skobeleva, K., Erokhin, M., Methner, A., Georgiev, P., et al. (2018). Presenilin-1 delta E9 mutant induces STIM1-driven store-operated calcium channel hyperactivation in hippocampal neurons. Mol. Neurobiol. 55, 4667-4680. doi: 10.1007/s12035-017-0674-4

Ryazantseva, M., Skobeleva, K., and Kaznacheyeva, E. (2013). Familial Alzheimer's disease-linked presenilin-1 mutation M146V affects storeoperated calcium entry: does gain look like loss? Biochimie 95, 1506-1509. doi: 10.1016/j.biochi.2013.04.009

Ryu, C., Jang, D. C., Jung, D., Kim, Y. G., Shim, H. G., Ryu, H. H., et al. (2017). STIM1 regulates somatic $\mathrm{Ca}^{2+}$ signals and intrinsic firing properties of cerebellar purkinje neurons. J. Neurosci. 37, 8876-8894. doi: 10.1523/JNEUROSCI.3973-16.2017

Salido, G. M., Sage, S. O., and Rosado, J. A. (2009). TRPC channels and store-operated $\mathrm{Ca}(2+)$ entry. Biochim. Biophys. Acta 1793, 223-230. doi: 10.1016/j.bbamcr.2008.11.001

Sanati, M., Khodagholi, F., Aminyavari, S., Ghasemi, F., Gholami, M., Kebriaeezadeh, A., et al. (2019). Impact of Gold Nanoparticles on amyloid $\beta$-induced Alzheimer's disease in a rat animal model: involvement of STIM proteins. ACS Chem. Neurosci. 10, 2299-2309. doi: 10.1021/acschemneuro.8b00622

Sather, W. A., and Dittmer, P. J. (2019). Regulation of voltage-gated calcium channels by the ER calcium sensor STIM1. Curr. Opin. Neurobiol. 57, 186-191. doi: 10.1016/j.conb.2019.01.019

Sattler, R., and Tymianski, M. (2000). Molecular mechanisms of calciumdependent excitotoxicity. J. Mol. Med. 78, 3-13. doi: 10.1007/s001090000077

Saul, S., Stanisz, H., Backes, C. S., Schwarz, E. C., and Hoth, M. (2014). How ORAI and TRP channels interfere with each other: interaction models and examples from the immune system and the skin. Eur. J. Pharmacol. 739, 49-59. doi: 10.1016/j.ejphar.2013.10.071

Segal, M., and Korkotian, E. (2014). Endoplasmic reticulum calcium stores in dendritic spines. Front. Neuroanat. 8:64. doi: 10.3389/fnana.2014.00064

Selvarai, S., Sun, Y., Sukumaran, P., and Singh, B. B. (2016). Resveratrol activates autophagic cell death in prostate cancer cells via downregulation of STIM1 and the mTOR pathway. Mol. Carcinog. 55, 818-831. doi: 10.1002/mc.22324

Selvaraj, S., Sun, Y., Watt, J. A., Wang, S., Lei, S., Birnbaumer, L., et al. (2012). Neurotoxin-induced ER stress in mouse dopaminergic neurons involves downregulation of TRPC1 and inhibition of AKT/mTOR signaling. J. Clin. Invest. 122, 1354-1367. doi: 10.1172/JCI61332

Serwach, K., and Gruszczynska-Biegala, J. (2019). STIM proteins and glutamate receptors in neurons: role in neuronal physiology and neurodegenerative diseases. Int. J. Mol. Sci. 20:2289. doi: 10.3390/ijms20092289

Sharma, S., Quintana, A., Findlay, G. M., Mettlen, M., Baust, B., Jain, M., et al. (2013). An siRNA screen for NFAT activation identifies septins as coordinators of store-operated $\mathrm{Ca}^{2+}$ entry. Nature 499, 238-242. doi: 10.1038/nature12229

Shinde, A. V., Motiani, R. K., Zhang, X., Abdullaev, I. F., Adam, A. P., González-Cobos, J. C., et al. (2013). STIM1 controls endothelial barrier 
function independently of Orail and $\mathrm{Ca}^{2+}$ entry. Sci. Signal. 6: ra18. doi: 10.1126/scisignal.2003425

Siddiqui, T. A., Lively, S., Vincent, C., and Schlichter, L. C. (2012). Regulation of podosome formation, microglial migration and invasion by $\mathrm{Ca}(2+)$ signaling molecules expressed in podosomes. J. Neuroinflammation 9:250. doi: 10.1186/1742-2094-9-250

Simpson, P. B., Challiss, R. A., and Nahorski, S. R. (1995). Neuronal $\mathrm{Ca}^{2+}$ stores: activation and function. Trends Neurosci. 18, 299-306. doi: 10.1016/0166-2236(95)93919-O

Singaravelu, K., Nelson, C., Bakowski, D., de Brito, O. M., Ng, S. W., Di Capite, J., et al. (2011). Mitofusin 2 regulates STIM1 migration from the $\mathrm{Ca}^{2+}$ store to the plasma membrane in cells with depolarized mitochondria. J. Biol. Chem. 286, 12189-12201. doi: 10.1074/jbc.M110.174029

Siu, C. R., Balsor, J. L., Jones, D. G., and Murphy, K. M. (2015). Classic and Golli Myelin Basic Protein have distinct developmental trajectories in human visual cortex. Front. Neurosci. 9:138. doi: 10.3389/fnins.2015.00138

Skibinska-Kijek, A., Wisniewska, M. B., Gruszczynska-Biegala, J., Methner, A., and Kuznicki, J. (2009). Immunolocalization of STIM1 in the mouse brain. Acta Neurobiol. Exp. 69, 413-428.

Soboloff, J., Rothberg, B. S., Madesh, M., and Gill, D. L. (2012). STIM proteins: dynamic calcium signal transducers. Nat. Rev. Mol. Cell Biol. 13, 549-565. doi: $10.1038 / \mathrm{nrm} 3414$

Soboloff, J., Spassova, M. A., Dziadek, M. A., and Gill, D. L. (2006a). Calcium signals mediated by STIM and Orai proteins-a new paradigm in inter-organelle communication. Biochim. Biophys. Acta 1763, 1161-1168. doi: 10.1016/j.bbamcr.2006.09.023

Soboloff, J., Spassova, M. A., Hewavitharana, T., He, L. P., Xu, W., Johnstone, L. S., et al. (2006b). STIM2 is an inhibitor of STIM1-mediated store-operated $\mathrm{Ca}^{2+}$ entry. Curr. Biol. 16, 1465-1470. doi: 10.1016/j.cub.2006.05.051

Soboloff, J., Spassova, M. A., Tang, X. D., Hewavitharana, T., Xu, W., and Gill, D. L. (2006c). Orail and STIM reconstitute store-operated calcium channel function. J. Biol. Chem. 281, 20661-20665. doi: 10.1074/jbc.C600126200

Somasundaram, A., Shum, A. K., McBride, H. J., Kessler, J. A., Feske, S., Miller, R. J., et al. (2014). Store-operated CRAC channels regulate gene expression and proliferation in neural progenitor cells. J. Neurosci. 34, 9107-9123. doi: 10.1523/JNEUROSCI.0263-14.2014

Song, I., and Huganir, R. L. (2002). Regulation of AMPA receptors during synaptic plasticity. Trends Neurosci. 25, 578-588. doi: 10.1016/S0166-2236(02)02270-1

Spät, A., and Szanda, G. (2017). The role of mitochondria in the activation/maintenance of SOCE: store-operated $\mathrm{Ca}^{2+}$ entry and mitochondria. Adv. Exp. Med. Biol. 993, 257-275. doi: 10.1007/978-3-319-57732-6_14

Steinbeck, J., Nadine, H., Opatz, J., Gruszczynska-Biegala, J., Schneider, L., Theiss, S., et al. (2011). Store-operated calcium entry modulates neuronal network activity in a model of chronic epilepsy. Exp. Neurol. 232, 185-194. doi: 10.1016/j.expneurol.2011.08.022

Sukumaran, P., Sun, Y., Vyas, M., and Singh, B. B. (2015). TRPC1-mediated $\mathrm{Ca}^{2}+$ entry is essential for the regulation of hypoxia and nutrient depletiondependent autophagy. Cell Death Dis. 6: e1674. doi: 10.1038/cddis.2015.7

Sun, S., Zhang, H., Liu, J., Popugaeva, E., Xu, N. J., Feske, S., et al. (2014). Reduced synaptic STIM2 expression and impaired store-operated calcium entry cause destabilization of mature spines in mutant presenilin mice. Neuron 82, 79-93. doi: 10.1016/j.neuron.2014.02.019

Sun, Y., Selvaraj, S., Pandey, S., Humphrey, K. M., Foster, J. D., Wu, M., et al. (2018). MPP + decreases store-operated calcium entry and TRPC1 expression in Mesenchymal Stem Cell derived dopaminergic neurons. Sci. Rep. 8:11715. doi: 10.1038/s41598-018-29528-x

Sun, Y., Zhang, H., Selvaraj, S., Sukumaran, P., Lei, S., Birnbaumer, L., et al. (2017). Inhibition of L-type $\mathrm{Ca}^{2+}$ channels by TRPC1-STIM1 complex is essential for the protection of dopaminergic neurons. J. Neurosci. 37, 3364-3377. doi: 10.1523/JNEUROSCI.3010-16.2017

Tellios, V., Maksoud, M. J. E., Xiang, Y. Y., and Lu, W. Y. (2020). Nitric oxide critically regulates purkinje neuron dendritic development through a metabotropic glutamate receptor type 1-mediated mechanism. Cerebellum 19, 510-526. doi: 10.1007/s12311-020-01125-7

Tong, B. C., Lee, C. S., Cheng, W. H., Lai, K. O., Foskett, J. K., and Cheung, K. H. (2016). Familial Alzheimer's disease-associated presenilin 1 mutants promote $\gamma$-secretase cleavage of STIM1 to impair store-operated $\mathrm{Ca}^{2+}$ entry. Sci. Signal. 9: ra89. doi: 10.1126/scisignal.aaf1371

Twine, N. A., Janitz, K., Wilkins, M. R., and Janitz, M. (2011). Whole transcriptome sequencing reveals gene expression and splicing differences in brain regions affected by Alzheimer's disease. PLoS ONE 6:e16266. doi: 10.1371/journal.pone.0016266

Ureshino, R. P., Erustes, A. G., Bassani, T. B., Wachilewski, P., Guarache, G. C., Nascimento, A. C., et al. (2019). The Interplay between $\mathrm{Ca}^{2+}$ signaling pathways and neurodegeneration. Int. J. Mol. Sci. 20:6004. doi: 10.3390/ijms20236004

Verkhratsky, A., and Parpura, V. (2014). Store-operated calcium entry in neuroglia. Neurosci. Bull 30, 125-133. doi: 10.1007/s12264-013-1343-x

Verkhratsky, A., Reyes, R. C., and Parpura, V. (2014). TRP channels coordinate ion signalling in astroglia. Rev. Physiol. Biochem. Pharmacol. 166, 1-22. doi: 10.1007/112_2013_15

Vig, M., Peinelt, C., Beck, A., Koomoa, D. L., Rabah, D., Koblan-Huberson, M., et al. (2006). CRACM1 is a plasma membrane protein essential for storeoperated $\mathrm{Ca}^{2+}$ entry. Science 312, 1220-1223. doi: 10.1126/science.1127883

Vigont, V., Kolobkova, Y., Skopin, A., Zimina, O., Zenin, V., Glushankova, L., et al. (2015). Both Orail and TRPC1 are involved in excessive store-operated calcium entry in striatal neurons expressing mutant huntingtin exon 1. Front. Physiol. 6:337. doi: 10.3389/fphys.2015.00337

Vlachos, A., Korkotian, E., Schonfeld, E., Copanaki, E., Deller, T., and Segal, M. (2009). Synaptopodin regulates plasticity of dendritic spines in hippocampal neurons. J. Neurosci. 29, 1017-1033. doi: 10.1523/JNEUROSCI.5528-08.2009

Walsh, C. M., Doherty, M. K., Tepikin, A. V., and Burgoyne, R. D. (2010). Evidence for an interaction between Golli and STIM1 in store-operated calcium entry. Biochem. J. 430, 453-460. doi: 10.1042/BJ20100650

Waltereit, R., and Weller, M. (2003). Signaling from cAMP/PKA to MAPK and synaptic plasticity. Mol. Neurobiol. 27, 99-106. doi: 10.1385/MN:27:1:99

Wang, G. X., and Poo, M. M. (2005). Requirement of TRPC channels in netrin1-induced chemotropic turning of nerve growth cones. Nature 434, 898-904. doi: 10.1038/nature03478

Wang, Y., Deng, X., Mancarella, S., Hendron, E., Eguchi, S., Soboloff, J., et al. (2010). The calcium store sensor, STIM1, reciprocally controls Orai and CaV1.2 channels. Science 330, 105-109. doi: 10.1126/science.1191086

Wei, D., Mei, Y., Xia, J., and Hu, H. (2017). Orail and Orai3 mediate storeoperated calcium entry contributing to neuronal excitability in dorsal root ganglion neurons. Front. Cell. Neurosci. 11:400. doi: 10.3389/fncel.2017.00400

Williams, R. T., Manji, S. S., Parker, N. J., Hancock, M. S., Van Stekelenburg, L., Eid, J. P., et al. (2001). Identification and characterization of the STIM (stromal interaction molecule) gene family: coding for a novel class of transmembrane proteins. Biochem. J. 357, 673-685. doi: 10.1042/0264-6021:3570673

Wong, A., Grubb, D. R., Cooley, N., Luo, J., and Woodcock, E. A. (2013). Regulation of autophagy in cardiomyocytes by $\operatorname{Ins}(1,4,5) \mathrm{P}(3)$ and $\operatorname{IP}(3)-$ receptors. J. Mol. Cell Cardiol. 54, 19-24. doi: 10.1016/j.yjmcc.2012.10.014

Wu, M. M., Buchanan, J., Luik, R. M., and Lewis, R. S. (2006). $\mathrm{Ca}^{2+}$ store depletion causes STIM1 to accumulate in ER regions closely associated with the plasma membrane. J. Cell Biol. 174, 803-813. doi: 10.1083/jcb.200604014

Wu, Y., Whiteus, C., Xu, C. S., Hayworth, K. J., Weinberg, R. J., Hess, H. F., et al. (2017). Contacts between the endoplasmic reticulum and other membranes in neurons. Proc. Natl. Acad. Sci. U.S.A. 114, E4859-E4867. doi: 10.1073/pnas.1701078114

Yang, J., Yu, J., Li, D., Yu, S., Ke, J., Wang, L., et al. (2017). Storeoperated calcium entry-activated autophagy protects EPC proliferation via the CAMKK2-MTOR pathway in ox-LDL exposure. Autophagy 13, 82-98. doi: 10.1080/15548627.2016.1245261

Yang, X., Jin, H., Cai, X., Li, S., and Shen, Y. (2012). Structural and mechanistic insights into the activation of Stromal interaction molecule 1 (STIM1). Proc. Natl. Acad. Sci. U.S.A. 109, 5657-5662. doi: 10.1073/pnas.1118947109

Yap, K. A., Shetty, M. S., Garcia-Alvarez, G., Lu, B., Alagappan, D., OhHora, M., et al. (2017). STIM2 regulates AMPA receptor trafficking and plasticity at hippocampal synapses. Neurobiol. Learn. Mem. 138, 54-61. doi: 10.1016/j.nlm.2016.08.007

Yuan, J. P., Zeng, W., Dorwart, M. R., Choi, Y. J., Worley, P. F., and Muallem, S. (2009). SOAR and the polybasic STIM1 domains gate and regulate Orai channels. Nat. Cell Biol. 11, 337-343. doi: 10.1038/ncb1842 
Zhang, C., and Thomas, D. W. (2016). Stromal Interaction Molecule 1 rescues store-operated calcium entry and protects NG115-401L cells against cell death induced by endoplasmic reticulum and mitochondrial oxidative stress. Neurochem. Int. 97, 137-145. doi: 10.1016/j.neuint.2016.04.002

Zhang, H., Sun, S., Wu, L., Pchitskaya, E., Zakharova, O., Fon Tacer, K., et al. (2016). Store-operated calcium channel complex in postsynaptic spines: a new therapeutic target for Alzheimer's disease treatment. J. Neurosci. 36, 11837-11850. doi: 10.1523/JNEUROSCI.1188-16.2016

Zhang, H., Wu, L., Pchitskaya, E., Zakharova, O., Saito, T., Saido, T., et al. (2015). Neuronal store-operated calcium entry and mushroom spine loss in amyloid precursor protein knock-in mouse model of Alzheimer's disease. J. Neurosci. 35, 13275-13286. doi: 10.1523/JNEUROSCI.1034-15.2015

Zhang, M., Song, J. N., Wu, Y., Zhao, Y. L., Pang, H. G., Fu, Z. F., et al. (2014). Suppression of STIM1 in the early stage after global ischemia attenuates the injury of delayed neuronal death by inhibiting storeoperated calcium entry-induced apoptosis in rats. Neuroreport 25, 507-513. doi: 10.1097/WNR.0000000000000127

Zhang, S. L., Yu, Y., Roos, J., Kozak, J. A., Deerinck, T. J., Ellisman, M. H., et al. (2005). STIM1 is a $\mathrm{Ca}^{2+}$ sensor that activates CRAC channels and migrates from the $\mathrm{Ca}^{2+}$ store to the plasma membrane. Nature 437, 902-905 doi: $10.1038 /$ nature04147

Zhou, Z., Xu, H., Liu, B., Dun, L., Lu, C., Cai, Y., et al. (2019). Suppression of lncRNA RMRP ameliorates oxygen-glucose deprivation/re-oxygenationinduced neural cells injury by inhibiting autophagy and PI3K/Akt/mTORmediated apoptosis. Biosci. Rep. 39:BSR20181367. doi: 10.1042/BSR20181367

Conflict of Interest: The authors declare that the research was conducted in the absence of any commercial or financial relationships that could be construed as a potential conflict of interest.

Copyright (C) 2020 Serwach and Gruszczynska-Biegala. This is an open-access article distributed under the terms of the Creative Commons Attribution License (CC BY). The use, distribution or reproduction in other forums is permitted, provided the original author(s) and the copyright owner(s) are credited and that the original publication in this journal is cited, in accordance with accepted academic practice. No use, distribution or reproduction is permitted which does not comply with these terms. 